\title{
High-energy limit of collision-induced false vacuum decay
}

\author{
Sergei Demidov and Dmitry Levkov \\ Institute for Nuclear Research of the Russian Academy of Sciences, \\ 60-th October Anniversary Prospect 7a, Moscow, 117312 Russia \\ E-mail: demidov@ms2.inr.ac.ru, levkov@ms2.inr.ac.ru
}

ABSTRACT: We develop a consistent semiclassical description of field-theoretic collisioninduced tunneling at arbitrary high collision energies. As a playground we consider a $(1+1)$-dimensional false vacuum decay initiated by a collision of $N$ particles at energy $E$, paying special attention to the realistic case of $N=2$ particles. We demonstrate that the cross section of this process is exponentially suppressed at all energies. Moreover, the respective suppressesion exponent $F_{N}(E)$ exhibits a specific behavior which is significant for our semiclassical method and assumed to be general: it decreases with energy, reaches absolute minimum $F=F_{\min }(N)$ at a certain threshold energy $E=E_{\mathrm{rt}}(N)$, and stays constant at higher energies. We show that the minimal suppression $F_{\min }(N)$ and threshold energy can be evaluated using a special class of semiclassical solutions which describe exponentially suppressed transitions but nevertheless evolve in real time. Importantly, we argue that the cross section at energies above $E_{\mathrm{rt}}(N)$ is computed perturbatively in the background of the latter solutions, and the terms of this perturbative expansion stay bounded in the infinite-energy limit. Transitions in the high-energy regime proceed via emission of many soft quanta with total energy $E_{\mathrm{rt}}$; the energy excess $E-E_{\mathrm{rt}}$ remains in the colliding particles till the end of the process.

KEYWORDS: Solitons Monopoles and Instantons, Nonperturbative Effects

ArXiv ePrint: 1503.06339 


\section{Contents}

1 Introduction 1

2 Perturbative expansion in the background of a bounce 5

$\begin{array}{lll}3 & \text { From Euclidean to real-time solutions } & 7\end{array}$

4 Real-time instantons 12

5 Transitions at $E>E_{\text {rt }} \quad 15$

6 Stability of perturbative expansion around the real-time instanton $\quad 18$

$\begin{array}{lll}7 & \text { Perturbative method at high energies } & 21\end{array}$

8 Summary and discussion $\quad 25$

A Multiparticle cross sections $\quad 26$

B High-frequency tail of the semiclassical solution 28

\section{Introduction}

Exponential suppression of probabilities precludes direct observation of extraordinary tunneling phenomena such as baryon number violation in instanton-like electroweak transitions [1-3] or spontaneous decay of allegedly false Higgs vacuum [4-8]. Quantum mechanical intuition suggests, however, that tunneling probabilities grow with energy. Indeed, tunneling phenomena of the above sort occur at higher rates $[9,10]$ in two-particle collisions:

$$
\sigma(E) \propto \mathrm{e}^{-F(E) / g^{2}},
$$

where the suppression exponent ${ }^{1} F(E)$ decreases with collision energy $E$, while $g$ is a small coupling constant. The central question is whether the exponential suppression in eq. (1.1) disappears at sufficiently high energies and, if it does not, what is the value of the suppression exponent at $E \rightarrow+\infty$.

This question is surprisingly nontrivial. Field-theoretic tunneling involves barriers of finite heights $E_{\mathrm{cb}}$ given by the energies of the critical bubble [12-15] and sphaleron [16, 17] in scalar and gauge theories, see figure 1a. Nevertheless, the respective collision-induced transitions cannot become unsuppressed ${ }^{2}$ at $E>E_{\mathrm{cb}}[20,21]$. Consider e.g. massless fermions $\psi$ and $\bar{\psi}$ coupled with small Yukawa constant $Y$ to the scalar sector of the theory. ${ }^{3}$ Their contribution to the scalar self-energy $\Pi\left(Q^{2}\right)$ obeys dispersion relation [21],

$$
\left.\frac{d^{2}}{\left(d Q^{2}\right)^{2}} \Pi\left(Q^{2}\right)\right|_{Q^{2} \rightarrow 0}=-\frac{8}{\pi Y^{2}} \int \frac{d E}{E} \sigma_{\text {tot }}(E)
$$

\footnotetext{
${ }^{1}$ Dubbed "holy grail function" [11].

${ }^{2}$ Gravitational interactions are completely different in this respect $[18,19]$.

${ }^{3}$ It is straightforward to generalize this argument to other setups.
} 


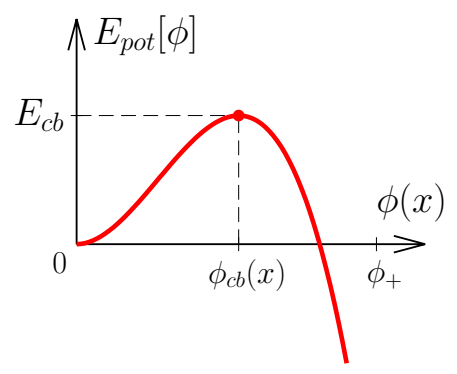

(a)

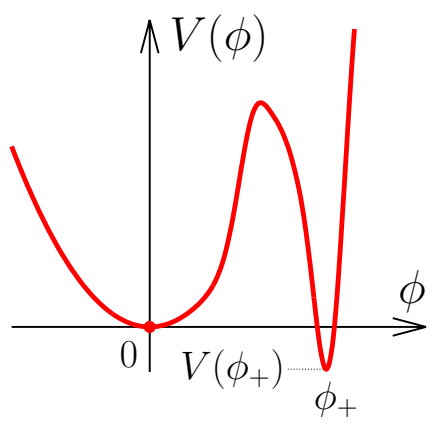

(b)

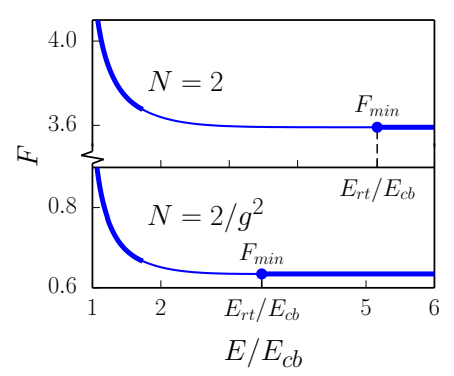

(c)

Figure 1. (a) A sketch of the potential barrier $E_{\text {pot }}[\phi]$ between the vacua $\phi=0$ and $\phi=\phi_{+}$. (b) Scalar potential $V(\phi)$. (c) Suppression exponent $F_{N}(E)$ at high energies.

where $\sigma_{\text {tot }}(E)$ is the total annihilation cross section $\psi \bar{\psi} \rightarrow$ anything at the center-of-mass energy $E$ and we ignore irrelevant scalar masses. If the fermion annihilation leads to unsuppressed over-barrier transitions at $E>E_{\mathrm{cb}}$, the self-energy $\Pi\left(Q^{2}\right)$ in eq. (1.2) receives large nonperturbative contributions at small $Q^{2}$. This would contradict the standard perturbation theory which is valid at low energies. Thus, the cross section (1.1) of collision-induced tunneling is exponentially suppressed at $E>E_{\mathrm{cb}}$.

In this paper we describe collision-induced tunneling at high energies. To be specific, we consider false vacuum decay in the $(1+1)$-dimensional scalar field model

$$
S[\phi]=\frac{1}{g^{2}} \int d^{2} x\left[\frac{1}{2}\left(\partial_{\mu} \phi\right)^{2}-V(\phi)\right]
$$

with false and true vacua at $\phi=0$ and $\phi=\phi_{+}$, respectively; the scalar potential $V(\phi)$ is shown in figure $1 \mathrm{~b}$. We work at weak coupling, $g \ll 1$. At zero energy the vacuum $\phi=0$ decays spontaneously via formation of an expanding bubble with $\phi \approx \phi_{+}$inside. Below we study the same decay accompanied by a collision of $N \phi$-quanta at energy $E$. We compute the suppression exponent $F_{N}(E)$ of the corresponding inclusive cross section at high energies.

Our numerical result ${ }^{4}$ for $F_{N}(E)$ is presented in figure 1c. This function decreases with energy, reaches minimum $F_{\min }(N)$ at $E=E_{\mathrm{rt}}(N)$ and stays constant at higher energies. With the aid of the Rubakov-Son-Tinyakov conjecture [22] we extrapolate results to the two-particle initial state and find similar behavior of the exponent $F(E) \equiv F_{2}(E)$ in eq. (1.1) (upper panel in figure 1c). We conclude that collision-induced false vacuum decay is exponentially suppressed at arbitrary high energies.

Energy-independent suppression exponent $F=F_{\min }$ of collision-induced tunneling at high energies was proposed in ref. [23] and observed in toy models of refs. [24, 25]. We find the same behavior in the full-fledged field-theoretic model.

Besides, we demonstrate that induced false vacuum decay at the threshold $E=E_{\mathrm{rt}}(N)$, despite being exponentially suppressed, is described by one-parametric family of complex

\footnotetext{
${ }^{4}$ In numerical calculations we use a specific form of $V(\phi)$ which is not significant at the moment.
} 
semiclassical solutions $\phi=\phi_{\mathrm{rt}}(x)$ evolving in real time $t \equiv x^{0}$. These solutions were introduced in ref. [26] under the name real-time instantons. They satisfy complexified classical field equations with certain boundary conditions in the asymptotic past and future. The initial particle number $N$ parametrizing the solutions enters the conditions at $t \rightarrow-\infty$. One can show [26] that the minimal suppression $F_{\min }(N)$ and respective energy $E_{\mathrm{rt}}(N)$ are computed as functionals on the real-time instantons.

We argue on general grounds that if the real-time instantons exist for some collisioninduced process, the respective suppression exponent is energy-independent and equal to $F_{\min }(N)$ at $E>E_{\mathrm{rt}}(N)$. Thus, finding the family of these solutions in a given model, one obtains the exponent $F_{N}(E)$ in the entire high-energy region $E>E_{\mathrm{rt}}(N)$.

Most importantly, we demonstrate that the real-time instantons serve as backgrounds for the long-awaited $[23,27]$ perturbative description of collision-induced tunneling at high energies, and the respective corrections are bounded in the high-energy limit. This remarkable feature is in sharp contrast to the properties of perturbative expansions in Euclidean backgrounds $[9,10]$ which blow up $[3,11,28]$ at $E \sim E_{\mathrm{cb}}$. Let us explain the difference by considering scatterings of particles at energy $\Delta E$ in different backgrounds. At a crude level the quantum particles can be regarded as small-amplitude high-frequency waves $\delta \phi \propto \mathrm{e}^{ \pm i \Delta E t}$ added to the background. In the Euclidean case $\delta \phi$ grows as $\mathrm{e}^{\Delta E \tau}$ with $\tau=-i t$, and nonlinear backreaction effects become essential at high $\Delta E$. In other words, the perturbative expansion in $\delta \phi$ breaks down. In the opposite case of the real-time instanton the waves $\delta \phi$ evolve adiabatically and do not change the soft background. Scattering of these waves can be described perturbatively.

Using the above observation, we propose a working perturbative scheme [29] for evaluating the inclusive cross section of collision-induced tunneling as series in $g^{2}$ in the most interesting case of high energies $E>E_{\mathrm{rt}}(2)$ and two initial particles. The receipt is as follows. One starts by developing a formal perturbation theory in the background of a real-time instanton $\phi_{\text {rt }}(x)$ with parameter $N=N_{0}$. Namely, one considers the $(n+2)$ point Green's function

$$
\mathcal{G}_{\mathrm{rt}} \equiv\left\langle\Psi_{\mathrm{rt}}\left|\phi\left(x_{1}\right) \ldots \phi\left(x_{n+2}\right)\right| \Psi_{0}\right\rangle=\int \mathcal{D} \phi \Psi_{\mathrm{rt}}^{*}[\phi] \phi\left(x_{1}\right) \ldots \phi\left(x_{n+2}\right) \mathrm{e}^{i S[\phi]} \Psi_{0}[\phi]
$$

between the false vacuum $\Psi_{0}$ and the most probable final state ${ }^{5} \Psi_{\mathrm{rt}}$ of this real-time instanton. One substitutes

$$
\phi(x) \equiv \phi_{\mathrm{rt}}(x)+g \delta \phi(x)
$$

into eq. (1.4) and evaluates the path integral over fluctuations $\delta \phi$ as series in $g$. The terms in these series depend on the auxiliary parameter $N_{0}$ of the real-time instanton which characterizes the background configuration. Using the LSZ reduction formula, one extracts from the Green's function the amplitude of the process $2 \rightarrow n+\Psi_{\mathrm{rt}}$, where the final state contains $n$ particles on top of $\Psi_{\mathrm{rt}}$. We argue that the final states of this kind form a complete set. Summing over them, one finds the inclusive cross section of the collision-induced process. The final result is obtained in the limit $N_{0} \rightarrow 0$.

\footnotetext{
${ }^{5} \mathrm{~A}$ coherent state to be specified in the main body of the paper.
} 
Our method is different from the standard instanton perturbation theory [9, 10] in several respects. First, we use the real-time instanton as a background. This guarantees stability of the perturbative expansion at high energies. Second, we introduce an additional parameter $N_{0}$ of $\phi_{\mathrm{rt}}$ and send $N_{0} \rightarrow 0$ in the end of calculations. Indeed, the real-time instanton with $N_{0}=0$, as we argue in the main body of the paper, is the saddle-point configuration for the path integral (1.4). Formally it describes transition from vacuum: two initial particles of the process are represented by two $\phi$-factors in the integrand of eq. (1.4) which do not affect the saddle-point solution. On the other hand, $\phi_{\mathrm{rt}}(t, x)$ turns out to be singular at $N_{0}=0$. We therefore work with smooth saddle-point configurations at $N_{0}>0$ and recover ${ }^{6}$ the correct results in the limit $N_{0} \rightarrow 0$. Third and finally, although below we evaluate only the leading inclusive suppression exponent $F=F_{\min }$ at $E>E_{\text {rt }}$, our perturbative approach can be used for the prefactor and exclusive cross sections at high energies.

With the help of the perturbative method we identify ${ }^{7}$ the dominant mechanism of the collisioninduced transitions at $E>E_{\mathrm{rt}}(2)$ and $N=2$, see figure 2. We observe that the colliding particles emit many soft quanta which form a bubble of true vacuum with energy $E_{\mathrm{rt}}(2)$; the energy excess $E-$ $E_{\mathrm{rt}}(2)$ remains in the initial particles till the end of the process.

From a general prospective our results support

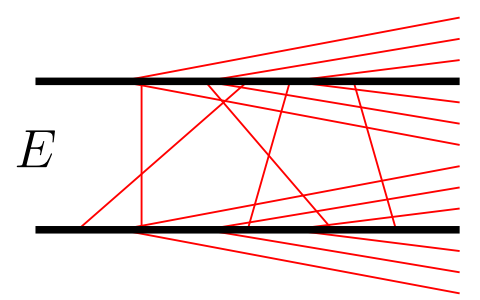

Figure 2. Transitions at $E>E_{\mathrm{rt}}(2)$. exponential suppression of collision-induced tunneling at arbitrary high energies and per se put on shaky ground proposed searches for nonperturbative phenomena at future colliders [32] or in cosmic ray events [33, 34], cf. ref. [35]. For example, it was found in refs. [36, 37] that the suppression exponent of electroweak baryon number violation in two-particle collisions is almost energy-independent at $E \sim 15 \mathrm{TeV}$. If the minimum $E=E_{\mathrm{rt}}(2)$ is somewhere near this point, the respective cross section is suppressed at all energies by a deadly factor $\mathrm{e}^{-F(15 \mathrm{TeV}) / \alpha_{W}} \sim 10^{-100}$, where $\alpha_{W}$ is the electroweak coupling and we took numerics from refs. [36, 37]. To get reasonable probabilities, one should consider models with tunneling rates raised by dynamical mechanisms [38-41], resort to strong coupling [42, 43] or exotica [44].

We argued in ref. [45] that the collision-induced false vacuum decay in $(1+1)$ dimensions turns into production of kink-like soliton pairs from particles once the energy densities of the two vacua are leveled, $V(0)-V\left(\phi_{+}\right) \rightarrow 0$. One expects that the properties indicated above hold in this limit. In particular, the cross section of creating a pair of solitons from two particles is exponentially small at all energies, and the suppression exponent of this process does not depend on energy above a certain threshold.

This paper is organized as follows. In section 2 we recall perturbative expansion in the background of a Euclidean bounce. This technique reproduces exponentially growing

\footnotetext{
${ }^{6}$ This makes our procedure a regularized version of Landau method [24, 30, 31] of singular semiclassical solutions.

${ }^{7}$ For simplicity we consider the process in a large finite volume.
} 
collision-induced cross section at low energies but breaks down at $E \gtrsim E_{\mathrm{cb}}$. We proceed with moderate energies in section 3 and demonstrate how the bounce at $E \ll E_{\mathrm{cb}}$ is connected with the real-time instantons at $E=E_{\mathrm{rt}}(N)>E_{\mathrm{cb}}$. The latter semiclassical solutions and transitions at $E>E_{\mathrm{rt}}(N)$ are considered in sections 4 and 5, respectively. In section 6 we show that the same term of perturbative expansion which led to exponential growth of the cross section with energy in perturbation theory around the bounce, gives subdominant and exponentially decreasing contribution in the background of the real-time instanton. We propose a working perturbative description of collision-induced tunneling at $N=2$ and high energies in section 7 . Our results are summarized in section 8 .

\section{Perturbative expansion in the background of a bounce}

Let us explain the difficulties with the collision-induced tunneling by reviewing its lowenergy description $[9,10]$ in the model (1.3). We will also introduce terminology and sharpen contrast with the high-energy transitions.

Spontaneous decay of false vacuum at $E=$ 0 is described by the celebrated bounce solution $[14,15] \phi_{\mathrm{b}}(x)$, see figure 3 . The latter has Euclidean and Minkowski parts representing nucleation of a true vacuum bubble and its expansion to infinite size. Note that the bounce is Lorentz-invariant i.e. depends on $x^{2} \equiv x_{\mu} x^{\mu}$. At large negative $x^{2}$ it satisfies the Klein-Gordon equation in the false vacuum and therefore behaves as

$$
\phi_{\mathrm{b}}(x) \rightarrow \frac{c_{\mathrm{b}}}{2 \pi} K_{0}\left(m \sqrt{-x^{2}}\right) \quad \text { as } \quad x^{2} \rightarrow-\infty .
$$

Here $m$ is the mass in the false vacuum $\phi=0$. In eq. (2.1) and below we exploit universal complex time $t \equiv x^{0}$ which is real and pure imaginary in the respective parts of the contour in figure 3 . Parameter $c_{\mathrm{b}}$ is related to the bubble size $R_{\mathrm{b}}: \phi_{\mathrm{b}}$ is of order 1 at $x \sim R_{\mathrm{b}}$. In the thin-wall limit $m R_{\mathrm{b}} \gg 1$ one obtains $c_{\mathrm{b}} \propto \mathrm{e}^{m R_{\mathrm{b}}}$, where the

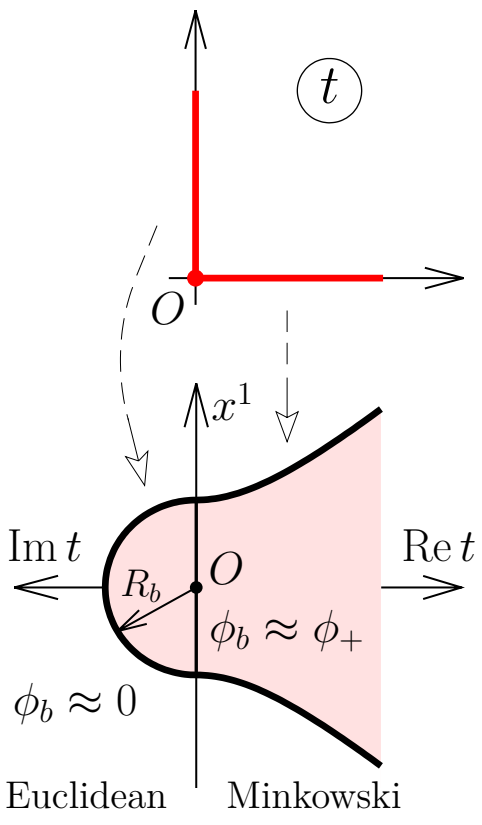

Figure 3. Bounce $\phi_{\mathrm{b}}(x)$. asymptotics of the Bessel function in eq. (2.1) was used.

To compute the amplitude of collision-induced tunneling, we consider the Green's function

$$
\begin{aligned}
\mathcal{G}_{b} & \equiv\left\langle\Psi_{\mathrm{b}}\left|\phi\left(x_{1}\right) \ldots \phi\left(x_{n+2}\right)\right| \Psi_{0}\right\rangle \\
& =\int \mathcal{D} \phi \Psi_{\mathrm{b}}^{*}[\phi] \phi\left(x_{1}\right) \ldots \phi\left(x_{n+2}\right) \mathrm{e}^{i S[\phi]} \Psi_{0}[\phi]
\end{aligned}
$$


where $\left|\Psi_{0}\right\rangle$ and $\left|\Psi_{\mathrm{b}}\right\rangle$ are the false vacuum and the final state of its decay at $E=0$, respectively. The latter describes expanding bubbles.

The idea $[9,10]$ is to evaluate the path integral in eq. (2.2) in the saddle-point approximation treating $\phi\left(x_{1}\right) \ldots \phi\left(x_{n+2}\right)$ as a prefactor. The relevant saddle-point configuration is the bounce $\phi_{\mathrm{b}}$. In the leading order one obtains,

$$
\mathcal{G}_{\mathrm{b}}=\mathcal{A}_{\mathrm{b}} \int d^{2} x_{0} \phi_{\mathrm{b}}\left(x_{1}-x_{0}\right) \ldots \phi_{\mathrm{b}}\left(x_{n+2}-x_{0}\right),
$$

where we introduced the bounce position $x_{0}$, ignored irrelevant saddle-point determinant and denoted the bounce amplitude by $\mathcal{A}_{\mathrm{b}}=\Psi_{\mathrm{b}}^{*}\left[\phi_{\mathrm{b}}\right] \mathrm{e}^{i S\left[\phi_{\mathrm{b}}\right]} \Psi_{0}\left[\phi_{\mathrm{b}}\right]$. Note that the action $S$ and zero-energy states $\Psi_{0}, \Psi_{\mathrm{b}}$ are translation-invariant ${ }^{8}$ and therefore independent of $x_{0}$. The bounce amplitude is computed in refs. $[14,15]$ : with exponential precision

$$
\left|\mathcal{A}_{\mathrm{b}}\right|^{2}=\mathrm{e}^{-2 \operatorname{Im} S\left[\phi_{\mathrm{b}}\right]},
$$

in particular, $\left|\Psi_{\mathrm{b}}\left[\phi_{\mathrm{b}}\right]\right|,\left|\Psi_{0}\left[\phi_{\mathrm{b}}\right]\right| \sim 1$. We remark that corrections to eq. (2.3) can be evaluated perturbatively using the bounce as a background: one substitutes $\phi=\phi_{\mathrm{b}}+g \delta \phi$ into the path integral and calculates it as series in $g^{2}$.

Importantly, the large- $x$ asymptotics of the bounce in eq. (2.1) reproduces correct residual at $k^{2}=m^{2}$ of its Fourier transform,

$$
\phi_{\mathrm{b}}(k) \equiv \int d^{2} x \mathrm{e}^{i k \cdot x} \phi_{\mathrm{b}}\left(x-x_{0}\right)=\frac{i c_{\mathrm{b}} \mathrm{e}^{i k \cdot x_{0}}}{k^{2}-m^{2}+i \epsilon}+\text { regular part },
$$

where we recalled that $K_{0}$ in eq. (2.1) is the Feynman propagator in two dimensions; we denote $k \cdot x \equiv k_{\mu} x^{\mu}$ and assume $\epsilon \rightarrow+0$. The residue $c_{\mathrm{b}}$ in eq. (2.5) is $k$-independent or "point-like" [3]. This property is specific to Euclidean solutions, as we argue below. One immediately obtains the $2 \rightarrow n$ transition amplitude from the LSZ formula,

$$
\mathcal{A}_{2 \rightarrow n}=\mathcal{A}_{\mathrm{b}}\left(\frac{c_{\mathrm{b}}}{g}\right)^{n+2}
$$

To derive this expression, we Fourier-transformed eq. (2.3), extracted the on-shell residues (2.5) and collapsed the integral over $x_{0}$ into the $\delta$-function ${ }^{9}$ representing the energymomentum conservation. Factors $g$ in eq. (2.6) compensate for non-canonical normalization of kinetic term in eq. (1.3).

The amplitude (2.6) exponentially grows with energy $E$ if the most probable final state contains $n \approx E / m$ nonrelativistic particles. To confirm this guess about the most probable state, we derive the inclusive cross section in appendix A,

$$
\sigma(E)=\sum_{n} \int\left|\mathcal{A}_{2 \rightarrow n}\right| d \Pi_{n}=\left|\mathcal{A}_{\mathrm{b}}\right|^{2} \int d^{2} \lambda \mathrm{e}^{i P \cdot \lambda+\frac{\left|c_{\mathrm{b}}\right|^{2}}{2 \pi g^{2}} K_{0}\left(m \sqrt{-\lambda^{2}+i \epsilon \lambda^{0}}\right)}
$$

\footnotetext{
${ }^{8}$ Here and below we assume asymptotic limits $t_{i, f} \rightarrow \mp \infty$ when all quantities oscillating with the initial $t_{i}$ or final $t_{f}$ times can be dropped.

${ }^{9}$ It is absorbed in the phase space volume, as usual.
} 
where the prefactors are ignored, $\Pi_{n}$ is the $n$-particle phase space volume and $P^{\mu}=(E, 0)$ is the total momentum in the center-of-mass frame. The variable $\lambda^{\mu}$ in eq. (2.7) can be regarded as a typical Compton wavelength of the final particles: the latter become nonrelativistic at $\lambda \gg m^{-1}$. At $g \ll 1$ the integral in eq. (2.7) is evaluated in the saddlepoint approximation. The extremum of the exponent is achieved at

$$
\lambda_{s}^{\mu}=(-2 i T, 0), \quad T=\frac{1}{2 m} \log \left(\frac{\left|c_{b}\right|^{2} \sqrt{m}}{2 g^{2} E \sqrt{4 \pi T}}\right),
$$

where the asymptotics of the Bessel function was used. This gives

$$
\sigma(E)=\mathrm{e}^{\left(2 T+m^{-1}\right) E-2 \operatorname{Im} S\left[\phi_{\mathrm{b}}\right]},
$$

see eq. (2.4). In the thin-wall limit $V\left(\phi_{+}\right) \rightarrow 0$ we substitute $c_{\mathrm{b}} \propto \mathrm{e}^{m R_{\mathrm{b}}}$ and obtain $T \approx R_{\mathrm{b}}$ with corrections proportional to $\log \left(m R_{\mathrm{b}}\right)$. The cross section $(2.9)$ in this limit coincides with that in refs. [46, 47], see also refs. [48-50].

The result (2.9) demonstrates exponential growth of the cross section with energy. It involves nonrelativistic final particles when $\lambda_{s} \sim T$ is large i.e. at $E \ll m \mathrm{e}^{2 m R_{\mathrm{b}}} / g^{2}$, where the exponent comes from $c_{\mathrm{b}}$. Moreover, the relativistic regime is never reached because at $E \gtrsim m / g^{2}$ weak coupling expansion becomes unreliable. Indeed, relative corrections to the leading-order result (2.3) are estimated ${ }^{10}[3]$ as $g^{2} n^{2}$, where $n^{2}$ comes from combinatorics; they are already large at $n \sim E / m \sim 1 / g^{2}$. One concludes that correct description of collision-induced tunneling at $E \gtrsim E_{\mathrm{cb}} \sim m / g^{2}$ should incorporate backreaction of the final-state particles on the semiclassical solution; we will pursue this approach in the next section.

Let us point at two specific features of the low-energy calculation. First, the bounce residue $c_{\mathrm{b}}$ in eq. (2.5) does not depend on $k$ as $k \rightarrow+\infty$. Second, the final state $\Psi_{\mathrm{b}}$ has zero energy and factorizes in eq. (2.3). We will see that these two properties do not hold for perturbative expansion about the relevant high-energy solutions.

\section{From Euclidean to real-time solutions}

We demonstrated that collision-induced transitions are no longer described by the bounce at $E \gtrsim E_{\mathrm{cb}}$. Since our interest lies in high energies, we set this background aside and search for true semiclassical solutions describing the false vacuum decay in the $N$-particle collisions. Consider the inclusive cross section,

$$
\sigma_{N}(E)=\sum_{\Psi_{i}, \Psi_{f}}\left|\left\langle\Psi_{f}\left|\hat{U}\left(t_{f}, t_{i}\right)\right| \Psi_{i} ; E, N\right\rangle\right|^{2} \approx \mathrm{e}^{-F_{N}(E) / g^{2}}
$$

where $\hat{U}$ is the evolution operator, $t_{i, f} \rightarrow \mp \infty$, we ignored the initial flux in the prefactor and introduced the suppression exponent $F_{N}(E)$ in the approximate equality. The sum in eq. (3.1) runs over all initial states $\Psi_{i}$ with energy $E$ and multiplicity $N$ in the false vacuum and final states $\Psi_{f}$ containing a bubble of true vacuum. Importantly, $\sigma_{N}(E)$ coincides at

\footnotetext{
${ }^{10}$ Sophisticated resummation $[51,52]$ shows that the true expansion parameter is $g^{2} n \ll 1$.
} 
$N=2$ with the two-particle collision-induced cross section $\sigma(E)$ and can be computed semiclassically at $N \gg 1$. Moreover, Rubakov-Son-Tinyakov conjecture [22] states that the suppression exponent $F_{N}(E)$ does not depend on $N$ at $N \ll 1 / g^{2}$, see refs. [53-56] for confirmations. This means that the two-particle exponent $F(E)$ in eq. (1.1) is obtained by extrapolating the semiclassical result for $F_{N}(E)$ to $g^{2} N \rightarrow 0$.

In direct semiclassical approach one writes a path integral for $\sigma_{N}(E)$ and evaluates it at $g \ll 1$ using the saddle-point configuration $\phi_{s}(t, x)$. In general, this configuration is complex. The saddle-point equations for $\phi_{s}$ are derived in ref. [22], see the summary in figure 4a. Like the bounce, this configuration satisfies the classical field equations ${ }^{11}$ $\delta S / \delta \phi=0$ or

$$
\left(\partial_{t}^{2}-\partial_{x}^{2}\right) \phi_{s}=-V^{\prime}\left(\phi_{s}\right)
$$

along the complex time contour $A B C D$ in figure 4 a. It contains an expanding bubble in the asymptotic future where the solution is real,

$$
\operatorname{Im} \phi_{s}, \quad \operatorname{Im} \partial_{t} \phi_{s} \rightarrow 0 \quad \text { as } \quad t \rightarrow+\infty .
$$

Peculiarities of $\phi_{s}(x)$ are related to the nontrivial initial state in eq. (3.1). Along the part $A B$ of the contour the saddle-point solution describes motion of the particles prior to the collision: in the asymptotic past it reduces to free waves

$$
\phi_{s} \rightarrow \int \frac{d k}{4 \pi \omega_{k}}\left(a_{k} \mathrm{e}^{-i k \cdot x}+b_{k}^{*} \mathrm{e}^{i k \cdot x}\right) \quad \text { as } \quad t \equiv x^{0} \rightarrow-\infty,
$$

where $k^{\mu}=\left(\omega_{k}, k\right), \omega_{k}^{2}=k^{2}+m^{2}$, and the limit is taken along the time contour of figure 4a. In eq. (3.2c) we introduced the classical counterparts $a_{k}, b_{k}^{*}$ of the annihilation and creation operators with relativistic normalization. They are related by the initial condition

$$
a_{k}=\mathrm{e}^{-2 \omega_{k} T-\theta} b_{k}
$$

involving two Lagrange multipliers $T$ and $\theta$ due to fixation of energy $E$ and the number $N$ of colliding particles. The latter quantities are given by the standard expressions

$$
g^{2} E=\int d k \frac{a_{k} b_{k}^{*}}{4 \pi}, \quad g^{2} N=\int d k \frac{a_{k} b_{k}^{*}}{4 \pi \omega_{k}} .
$$

In the limit $T \rightarrow+\infty$ eq. (3.2d) reduces to vacuum condition $a_{k}=0$ which corresponds to $E=N=0$. In this case $\phi_{s}(x)$ coincides with the bounce solution $\phi_{\mathrm{b}}(x)$. At finite $T$ and $\theta$ the saddle-point solution describes transition at nonzero $E$ and $N$. In what follows we solve equations (3.2) and relate $(T, \theta)$ to $(E, N)$ by eq. (3.3).

Given the saddle-point configuration $\phi_{s}(x)$, one evaluates the suppression exponent [22]

$$
F_{N}(E)=g^{2}\left(2 \operatorname{Im} S\left[\phi_{s}\right]-2 E T-N \theta\right)+\left.\operatorname{Im} \int d x \phi_{s} \partial_{t} \phi_{s}\right|_{t=t_{i}},
$$

where the last three terms are the initial-state contributions. Importantly, the method of Lagrange multipliers implies Legendre transform

$$
\partial_{E} F_{N}(E)=-2 g^{2} T, \quad \partial_{N} F_{N}(E)=-g^{2} \theta,
$$

which demonstrates that $T$ and $\theta$ are proportional to the derivatives of $F_{N}(E)$.

\footnotetext{
${ }^{11}$ At the spatial boundary we impose the standard energy-conserving condition $\partial_{x} \phi_{s} \rightarrow 0$ at $x \rightarrow \pm \infty$.
} 


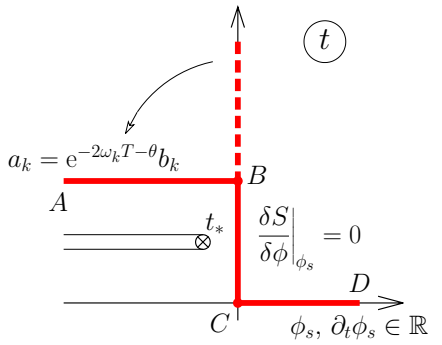

(a)

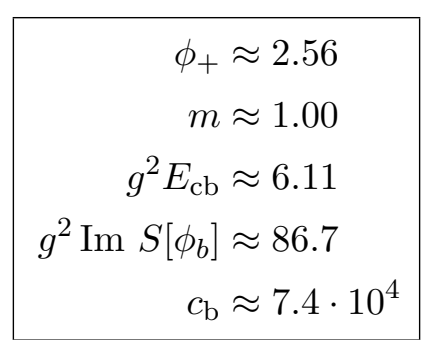

(b)

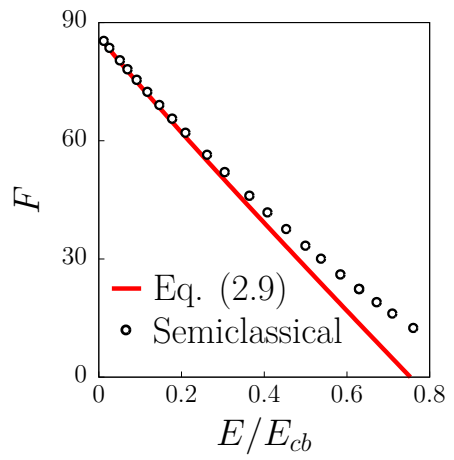

(c)

Figure 4. (a) Contour in complex time for $\phi_{s}(t, x)$. Saddle-point equations and boundary conditions at $t \rightarrow \pm \infty$ are written near the respective parts of the contour. The nearest singularity $t_{*}$ of the solution is marked by the crossed circle attached to the branch cut (thin double line). (b) Physical quantities in the model (3.6). (c) Suppression exponent at low energies.

We solve the boundary value problem (3.2) for different $T$ and $\theta$ numerically. To this end we specify the scalar potential in dimensionless units,

$$
V(\phi)=\frac{\phi^{2}}{2}\left[1-v W\left(\frac{\phi-2}{u}\right)\right],
$$

where $W(x)=\mathrm{e}^{-x^{2}}\left(x+x^{3}+x^{5}\right), u=0.4$, and the energy density $V\left(\phi_{+}\right)=-0.4$ of the true vacuum is set by tuning $v \approx 0.84$. Function (3.6) is plotted in figure $1 \mathrm{~b}$. It is almost quadratic at $\phi<\phi_{+} / 2$ and nontrivial at larger $\phi$, so that waves in eq. (3.2c) remain linear ${ }^{12}$ almost up to their collision point.

We numerically computed the physical quantities for the potential (3.6), see figure 4b. To this end we have found the bounce $\phi_{\mathrm{b}}(t, x)$ and the critical bubble $\phi_{\mathrm{cb}}(x)$, see ref. [15] for details. Recall that the bounce action $2 \operatorname{Im} S\left[\phi_{\mathrm{b}}\right]$ in eq. (2.4) is the suppression exponent of false vacuum decay at zero energy, whereas the energy $E_{\mathrm{cb}}$ of the critical bubble gives the height of the potential barrier between the vacua. We also extracted the bounce residue $c_{\mathrm{b}}$ from the asymptotics of $\phi_{\mathrm{b}}(t, x)$ at $x_{\mu} x^{\mu} \rightarrow-\infty$, see eq. (2.1). We remind that the coupling constant $g \ll 1$ scales out in the semiclassical calculations, cf. eq. (1.3).

We discretize equations (3.2) and introduce uniform $N_{t} \times 2 N_{x}$ lattice with sites $t_{i}$ and $x_{j}$ covering the contour $A B C D$ and space interval ${ }^{13}(-L, L), L=7$; the spatial lattice spacing is $\Delta x \equiv L / N_{x}$. We use the second-order finite-difference approximation for the field equation and trade Fourier transform in eq. (3.2c) for its discrete version. This turns the semiclassical boundary value problem into a set of $N_{t} \times 2 N_{x}$ nonlinear algebraic equations $^{14}$ for $\phi_{i j}=\phi_{s}\left(t_{i}, x_{j}\right)$ which are solved by the Newton-Raphson method [57]. Detailed description of our numerical technique will be presented elsewhere [58], see refs. [36, 59] for related works. In the subsequent sections we will concentrate on numerical solutions at

\footnotetext{
${ }^{12}$ Long nonlinear evolution in other models is costly for numerical computations.

${ }^{13}$ Larger intervals are used at low energies due to larger sizes of the respective solutions.

${ }^{14}$ The solutions are $P$-symmetric, $\phi_{s}(t, x)=\phi_{s}(t,-x)$, so we use only a half of the lattice with $x_{j}>0$.
} 
high energies and small multiplicities. We will need large $N_{t}$ and $N_{x}$ because the typical frequencies $\omega_{k} \sim E / N$ of these solutions are high. In particular, lattices $N_{t} \times N_{x}=3000 \times 150$ and $11000 \times 4000$ are required to reach acceptable numerical precision at $E \sim E_{\mathrm{cb}}$ and the highest energies, respectively.

Our interest lies in the semiclassical results at high energies and relatively small $N$. The well-known saddle-point configuration, however, is the bounce $\phi_{\mathrm{b}}(t, x)$ which satisfies the boundary value problem (3.2) at $T=+\infty, \theta=0$ and $E=N=0$. We continuously relate it to the high-energy solutions. Namely, we find solutions at large $T$ by iterative numerical method using the bounce as the zeroth-order approximation. After that we change $T$ and $\theta$ in small steps and obtain one slightly deformed solution $\phi_{s}(t, x)$ of eqs. (3.2) at each step. We compute the energy $E$ and initial particle number $N$ for every $\phi_{s}(t, x)$ by eqs. (3.3). An exemplary set of numerical solutions is marked by points $(E, N)$ in the central plot of figure 5. It was obtained by decreasing $T$ at $\theta=0$ (red/dark points), then increasing $\theta$ to $\theta=0.4$, and finally decreasing $T$ (green/light points) until the solution with $T=0$ and $\theta=0.4$ is reached (squared point). This procedure brings us to the high-energy region of interest. The other solutions in this region (not shown in figure 5) are obtained in similar manner, by changing $(T, \theta)$ in small steps and numerically solving the semiclassical equations (3.2).

Solutions $\phi_{s}=\phi_{\mathrm{rt}}(t, x)$ with $T=0$ and arbitrary $\theta$ are called "real-time instantons". A representative solution of this type corresponds to the squared point in figure 5 . In the next section we will find that the real-time instantons are radically different from the solutions with $T \neq 0$. We will develop an adequate high-energy description of collisioninduced tunneling based on their properties.

At low energies our semiclassical solutions reproduce the results of the previous section. Indeed, figure $4 \mathrm{c}$ compares the semiclassical exponent (3.4) at $\theta=0$ (points) with the exponent in eq. (2.9) (line), where the numerical values in figure $4 \mathrm{~b}$ are used. The two graphs coincide at $E \ll E_{\mathrm{cb}}$ despite the fact that the perturbative results are obtained at $N=2$ whereas the semiclassical solutions involve large multiplicities $N \sim 1 / g^{2}$, see figure 5. This is because $F_{N}(E)$ is independent ${ }^{15}$ of $N$ at $E<E_{\mathrm{cb}}$ [46], so we do not have to continue it to $g^{2} N \rightarrow 0$. Importantly, the perturbative graph in figure $4 \mathrm{c}$ becomes negative at $E \approx 0.8 E_{\mathrm{cb}}$ indicating apparent violation of unitarity. At these energies the perturbative expansion of section 2 is not reliable unlike the semiclassical method of this section.

Our numerical results show a dramatic change in the form of the saddle-point solutions at $E \approx E_{\mathrm{cb}}$. The low-energy solutions in figures $5 \mathrm{a}, 5 \mathrm{~b}$ resemble the bounce: they contain long Euclidean parts and describe creation of true vacuum bubbles (the latter are shown by red (dark) in the figures). Waves in the left parts of the plots represent initial particles. In contrast, at $E>E_{\mathrm{cb}}$ the initial waves are sharper and the bubbles are smaller, see figures $5 \mathrm{c}, 5 \mathrm{~d}$. Another property of high-energy solutions is small durations of their Euclidean evolutions and, nevertheless, nonzero values of the suppression exponents ${ }^{16}$ (3.4) thanks

\footnotetext{
${ }^{15}$ In the thin-wall approximation which works well [45] at $V\left(\phi_{+}\right)=-0.4$.

${ }^{16}$ Exponentially suppressed transitions at $E>E_{\mathrm{cb}}$ are called "dynamical tunneling" [60,61] to distinguish from the potential tunneling at low energies.
} 
(a)

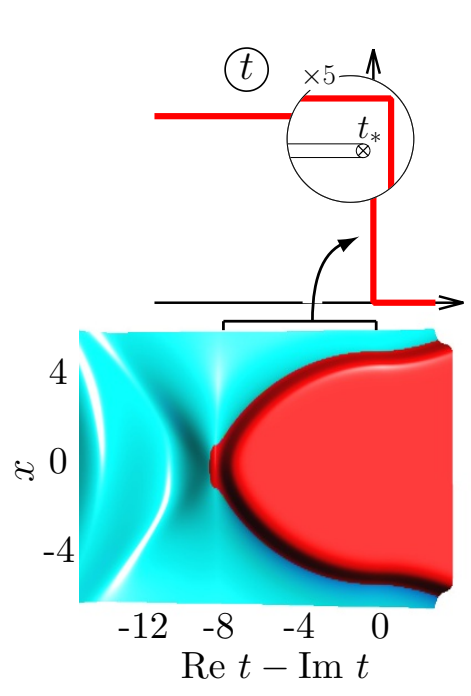

(b)

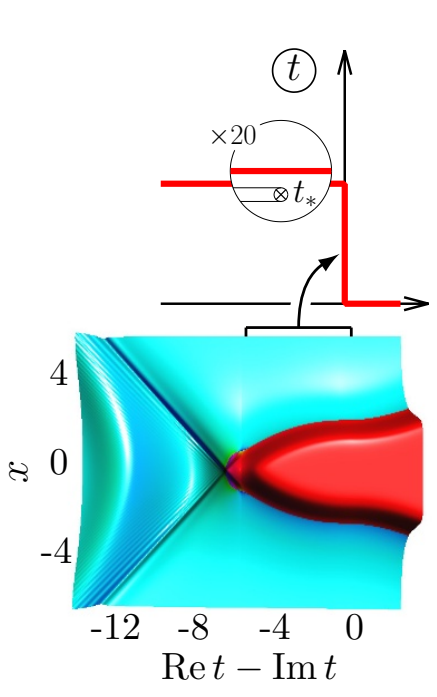

(c)
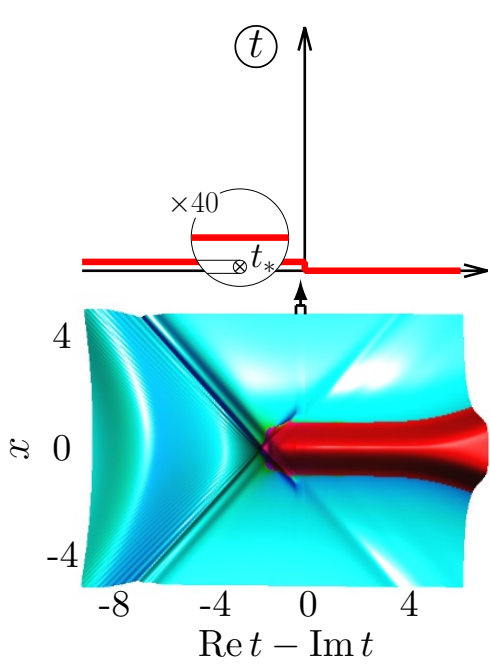

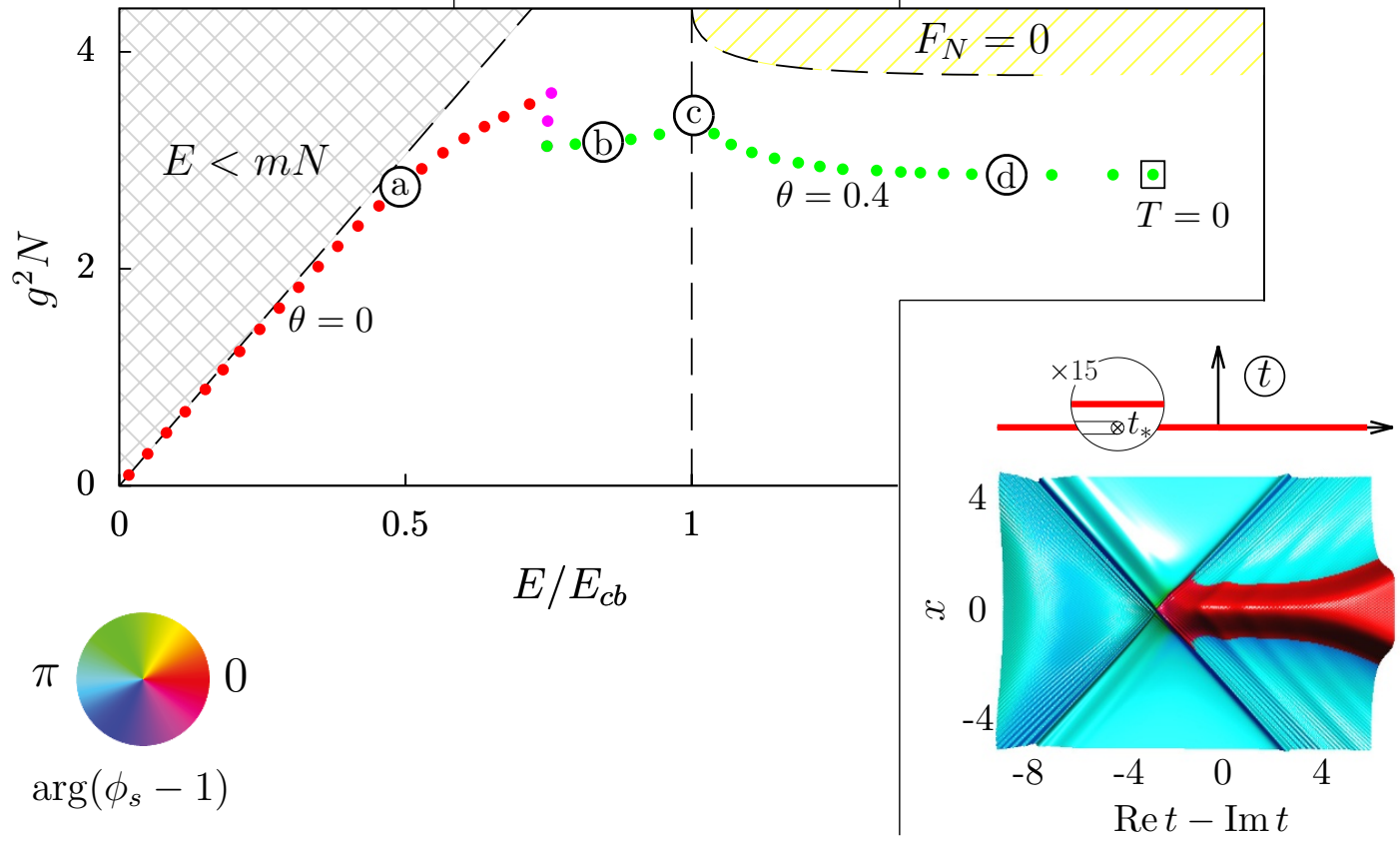

(d)

Figure 5. Central plot: $(E, N)$ plane of initial data. Parameters of the numerical solutions are indicated by red (dark gray), purple and green (light gray) points. Circles with letters represent solutions from the insets (a)-(d). The squared point corresponds to the real-time instanton with $T=0$ and $\theta=0.4$. Insets $(a)-(d)$ : Three-dimensional plots of the numerical solutions $\operatorname{Re} \phi_{s}(t, x)$, where the abscisses $\operatorname{Re} t-\operatorname{Im} t$ parametrize the contours in the complex time plane. Color shows $\arg \left(\phi_{s}-1\right)$. Euclidean parts of the solutions are marked by the square brackets with arrows. Above each $3 \mathrm{D}$ plot we draw the respective contour in the complex time plane, where the zoomed circular area with magnification factor displays the vicinity of the nearest singularity (crossed circle attached to the branch cut). The lattice size of all solutions is $N_{t} \times N_{x}=3000 \times 150$.

to complex-valued $\phi_{s}(t, x)$. In particular, the real-time instanton at $T=0$ evolves entirely along $t \in \mathbb{R}$. We will argue that this feature guarantees stable perturbative expansion at high energies. 
One wonders how the durations of Euclidean evolutions can be of any physical meaning: they are not even explicitly specified in figure 4a. Indeed, one expects that $\phi_{s}(t, x)$ are analytic functions of time and can be continued to any contours. But in fact, the solutions and, in particular, the bounce have branch-cut singularities starting at $t=t_{*}$ which separate their time contours from the real time axis, see figure $4 \mathrm{a}$. We compute positions of these singularities for the solutions (a)-(d) in figure 5. To this end we continue $\phi_{s}(t, x)$ to complex $t$ and find the points $t=t_{*}$ where $\left|V\left(\phi_{s}\right)\right|=\infty$, see the zoomed areas in the respective $t$-planes. The singularities prevent us from continuing the low-energy solutions to the real time axis. We find, however, that $\operatorname{Im} t_{*}$ decreases with energy and becomes negative for the solution (d) in figure 5 . The latter solution can be considered in real time, as well as the real-time instanton at $T=0$.

We find the semiclassical solutions at $g^{2} N=2$ (not shown in figure 5) and plot the respective suppression exponent $F_{N}(E)$ in figure 1c (thick line in the left part of the lower panel). The latter exponent monotonously decreases with energy until the point $T=0$ and $E=E_{\mathrm{rt}}(N)$ is reached. ${ }^{17}$ The region of few-particle initial states $N \ll 1 / g^{2}$ cannot be directly addressed at the present-day computers. So, we extrapolate numerical results into this region. Since $\mathrm{e}^{-\theta}$ analytically enters the semiclassical equations (3.2), the particle number $N$, action $S\left[\phi_{s}\right]$, and the last term in eq. (3.4) have regular Taylor expansions in this parameter. Moreover, $\mathrm{e}^{-\theta} \rightarrow 0$ leads to Feynman boundary conditions at $t \rightarrow-\infty$ and therefore to $g^{2} N \rightarrow 0$, see eq. (3.2d). Substituting all Taylor expansions into eq. (3.4) and using eq. (3.5), one finds that

$$
F_{N}(E)+g^{2} \theta N+g^{2} N=F(E)+O\left(g^{4} N^{2}\right) .
$$

We fit numerical data for the left-hand side of this equality with function $F(E)+d(E) \cdot g^{4} N^{2}$ and obtain the suppression exponent $F(E)$ (solid line in the left upper part of figure 1c). Numerical error of this procedure is expected to be smaller than 5\%. Our results show that the two-particle exponent $F(E)$ is also a decreasing function of energy.

We finish this section by remarking that the above semiclassical solutions describe classically forbidden transitions from the initial states with relatively small multiplicities $N$. Increasing $N$, one reaches the states decaying classically (region " $F_{N}=0$ " in figure 5). Classical transitions in the model (3.6) were studied in ref. [62], see refs. [38-40] for the related work.

\section{Real-time instantons}

The real-time instantons, i.e. solutions of the saddle-point equations at $T=0$ and arbitrary $\theta$, are special in many respects (see one of them in figure 6a). At a given $N$ they have the highest energies $E=E_{\mathrm{rt}}(N)$ and smallest suppression exponents $F_{\min }(N) \equiv F_{N}\left(E_{\mathrm{rt}}(N)\right)$ considered so far. Indeed, all solutions at smaller energies have positive parameter $T$ : we obtained them by lowering $T$ from $T=+\infty$ to $T=0$. Since $\partial_{E} F_{N}=-2 g^{2} T$, the exponent

\footnotetext{
${ }^{17}$ Data lines are not shown at high energies where the numerical errors are large. To decrease the computational cost, we find the solution at $E=E_{\mathrm{rt}}$ at high precision (next section) and fill the gap with thin interpolating line.
} 


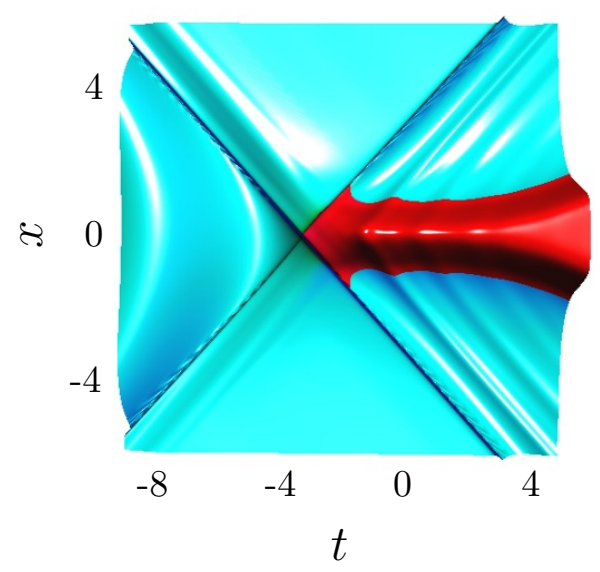

(a)

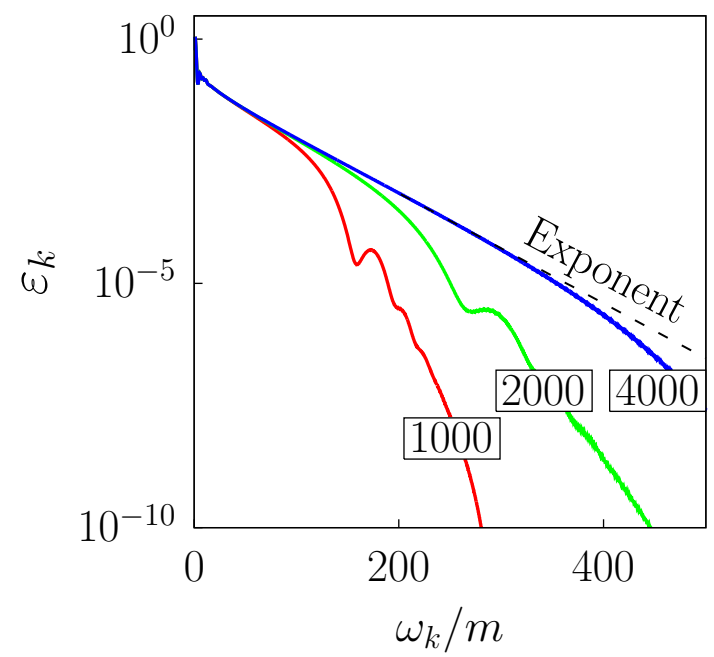

(b)

Figure 6. (a) Real-time instanton $\phi_{\mathrm{rt}}(t, x)$ and (b) its initial energy distributions $\varepsilon_{k}$ at different $N_{x}$ (numbers in boxes). In both figures $\theta=0.199,(E, N) \approx\left(2.4 E_{\mathrm{cb}}, 3.5 / g^{2}\right), N_{t}=11000$. Figure (a) uses colors of figure 5 and $N_{x}=2000$.

$F_{N}(E)$ decreases with energy and reaches a local minimum at $E=E_{\mathrm{rt}}(N)$. In section 5 we will argue that this minimum is global by showing that the suppression exponent is energy-independent at $E>E_{\mathrm{rt}}(N)$.

The above property implies that each real-time instanton describes collision-induced transitions from the states with fixed multiplicity $N$ and arbitrary energies. Indeed, the probability

$$
\sigma_{N}^{\max }=\int_{0}^{\infty} d E \mathrm{e}^{-F_{N}(E) / g^{2}}
$$

receives dominant contribution near the saddle point $E=E_{\text {rt }}(N)$ corresponding to the realtime instanton. One finds $\sigma_{N}^{\max } \approx \mathrm{e}^{-F_{\min }(N) / g^{2}}$, where $F_{\min }(N)$ is computed using $\phi_{\mathrm{rt}}(t, x)$.

In the previous section we obtained the real-time instantons from the larger family of semiclassical solutions with arbitrary $T$ and $\theta$. One can compute $\phi_{\text {rt }}$ directly by solving the semiclassical equations $(3.2 \mathrm{a}),(3.2 \mathrm{~b})$ with the initial condition

$$
a_{k}=\mathrm{e}^{-\theta} b_{k}
$$

cf. eq. (3.2d). Then the parameter $N$, energy $E_{\mathrm{rt}}(N)$ and minimal suppression exponent $F_{\min }(N)$ are given by the standard expressions (3.3), (3.4).

A convenient and important feature of the real-time instantons came as a surprising numerical fact in figure 5: they are defined along the real time axis $t \in \mathbb{R}$. Technically, this property is related to the initial condition (3.2d) and its consequence (4.1). Indeed, the positive- and negative-frequency terms in the integrand of eq. (3.2c) are of order $\tilde{b}_{k}^{*} \equiv$ $b_{k}^{*} \mathrm{e}^{-\omega_{k} T_{A B}}$ and $\tilde{a}_{k} \equiv a_{k} \mathrm{e}^{\omega_{k} T_{A B}}$, where $T_{A B}=\operatorname{Im} t_{A B}$ is the height of the time contour. The integrals of these terms in eq. (3.2c) converge faster and slower at higher $T_{A B}$, respectively. At $T_{A B}=T$ the terms are of the same order because $\tilde{a}_{k}=\mathrm{e}^{-\theta} \tilde{b}_{k}$ due to the initial 
condition (3.2d). This "optimal" contour lies right in the middle between the singularities of the solution: at somewhat higher or lower $T_{A B}$ one of the integrals in eq. (3.2c) diverges signaling that the contour hits the singularity. We use $T_{A B}=T$ in numerical calculations and show the corresponding contour in figures $5 \mathrm{a}-5 \mathrm{~d}$. At $T \rightarrow 0$ the optimal contour coincides with the real time axis.

The above argument turns quantitative if we use the high-frequency asymptotics of the solution. Namely, consider the energy of modes with the wave number $k$ in eq. (3.3),

$$
\varepsilon_{k} \equiv \frac{a_{k} b_{k}^{*}}{4 \pi}, \quad g^{2} E=\int d k \varepsilon_{k} .
$$

In appendix B we demonstrate that any smooth solution has exponential asymptotics,

$$
\varepsilon_{k} \rightarrow \varepsilon_{0} \mathrm{e}^{-2 \omega_{k} T_{*}} \quad \text { as } \quad k \rightarrow+\infty,
$$

where $T_{*}$ is a parameter of the solution. Extracting $a_{k}$ and $b_{k}^{*}$ from eq. (4.3) and initial condition (3.2d), one finds that the integral in eq. (3.2c) converges at $\left|T_{A B}-T\right|<T_{*}$ and diverges otherwise. Thus, $T_{*}$ is the distance from the optimal contour to the nearest singularity of the solution, $T_{*}=\left|T-\operatorname{Im} t_{*}\right|$.

In figure $6 \mathrm{~b}$ we plot energy distributions $\varepsilon_{k}$ for the real-time instanton with $\theta=0.199$ at different $N_{x}$. At larger $N_{x}$ and $\omega_{k} \gg m$ the graphs approach eq. (4.3) with $T_{*} \approx 0.013$. This indicates that the continuum limit of our numerical solution is a smooth configuration with singularities at finite distances $T_{*}$ from the real time axis.

Note that the latter fact is important because our conclusions about existence and properties of the real-time instantons rely on numerical calculations. In figure 7 we study the continuum limit in more detail. If $\phi_{\mathrm{rt}}(t, x)$ are smooth configurations, the numerical errors are expected to be polynomials in $\delta \equiv N_{x}^{-2} \propto(\Delta x)^{2}$ and $N_{t}^{-2}$ because we use the second-order finite-difference methods. This is indeed the case: lattice values of $E, N$ and $F_{\min }$ (points in figure 7 ) are not sensitive to $N_{t}$ and well approximated by quadratic functions of $\delta$ (lines). At large $N_{x}$ all numerical errors are proportional to $\delta$, see the inset in figure 7a. Once again we conclude that our lattice solutions with $T=0$ have well-defined continuum limits.

Numerical results for the minimal suppressions $F_{\min }(N)$ and respective energies $E_{\mathrm{rt}}(N)$ are shown in figure 8 . Dashed lines in figure $8 \mathrm{~b}$ are the lattice results, ${ }^{18}$ while the solid lines represent continuum limits obtained by quadratic extrapolations in $\delta=N_{x}^{-2}$ to $\delta=0$, cf. figure 7. Points $E=E_{\mathrm{rt}}$ in figure 1c are extracted from figure 8. In particular, we obtain results for the few-particle initial states $g^{2} N \ll 1$ by extrapolating $E_{\mathrm{rt}}(N)$ to $g^{2} N=0$ with linear function, and $F_{\mathrm{rt}}(N)$ by the method described in the end of section 3, see the dotted lines in figure 8 . The accuracy of our result for $F_{\mathrm{rt}}(0)$ is better than $5 \%$, while extrapolation of energy should be considered as illustrative. In particular, we cannot completely exclude the possibility that $E_{\mathrm{rt}} \rightarrow+\infty$ at $g^{2} N \rightarrow 0$. Nevertheless, it is likely that the point $E=E_{\mathrm{rt}}$ exists for the few-particle initial states, since it does for the multiparticle ones, cf. refs. $[25,26]$.

\footnotetext{
${ }^{18}$ In figure $8 \mathrm{a}$ they are indistinguishable from the continuum limit.
} 


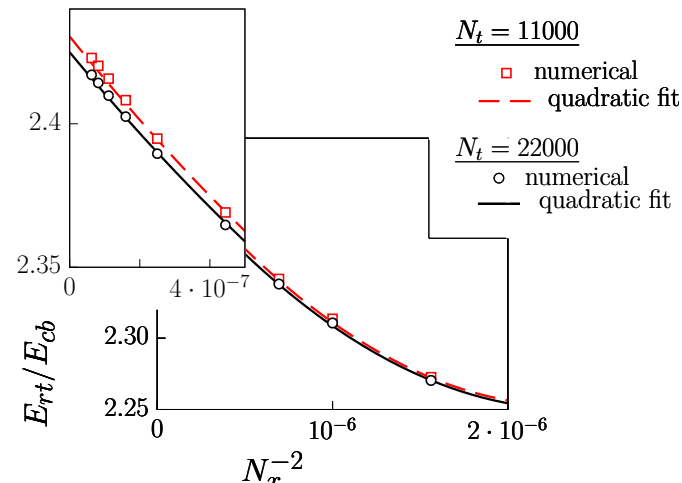

(a)

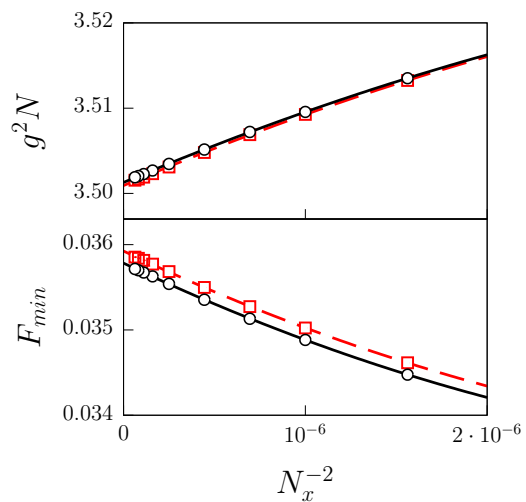

(b)

Figure 7. Continuum limits $N_{x} \rightarrow+\infty$ of the (a) real-time instanton energy $E_{\mathrm{rt}}$, (b) its initial particle number $N$ and suppression exponent $F_{\min } ; \theta=0.199$. We fit the data points with quadratic functions of $\delta \equiv N_{x}^{-2}$ (lines) in the range $N_{x}=800 \div 4000$.

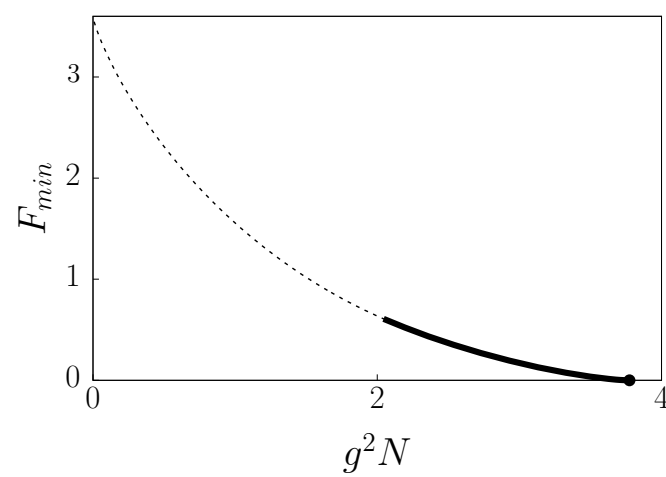

(a)

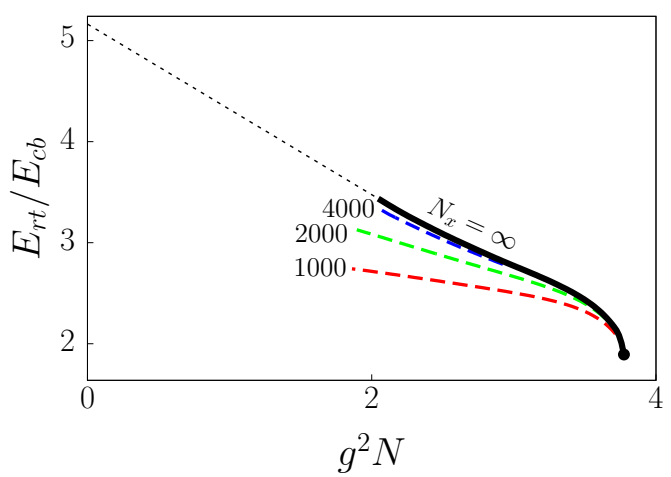

(b)

Figure 8. (a) Suppression exponent $F_{\min }(N)$ and (b) energy $E_{\mathrm{rt}}(N)$ of the real-time instantons. Dashed lines in figure (b) are the lattice results at $N_{t}=11000$ and different $N_{x}$ (numbers near the lines). Thick solid lines are obtained by extrapolating results to $N_{x} \rightarrow+\infty$.

\section{Transitions at $E>E_{\mathrm{rt}}$}

Numerical solutions at $E>E_{\mathrm{rt}}(N)$ look similar to the real-time instantons, cf. figures $9 \mathrm{a}$ and 6a. But in fact they are fundamentally different: we are going to demonstrate that they do not have continuum limits and lead to energy-independent suppression exponent $F_{N}(E)=F_{\min }(N)$.

To begin with, we find that the lattice values of the parameter $T$ monotonously decrease with energy and become negative at $E>E_{\mathrm{rt}}(N)$, see figure $9 \mathrm{~b}$. Then eq. (3.5) implies that the exponent $F_{N}(E)$ reaches minimum at $E=E_{\mathrm{rt}}(N)$ and increases at higher energies. The last feature, however, is not expected in continuum models. Indeed, the sum in the definition (3.1) of $F_{N}(E)$ runs over all initial states with energy $E$ and multiplicity $N$. These include, in particular, the state where $N-1$ particles perform transition at smaller 


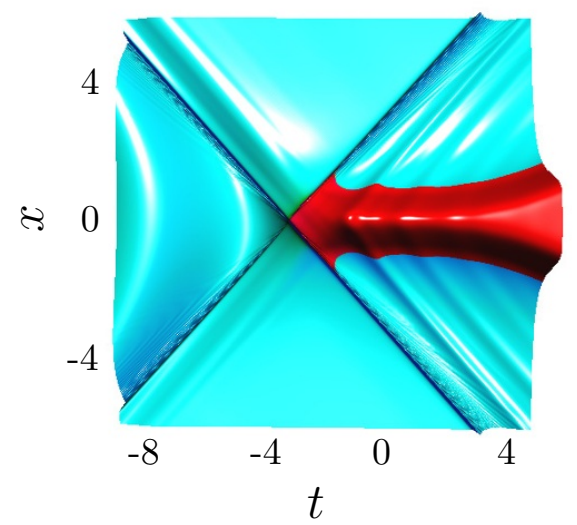

(a)

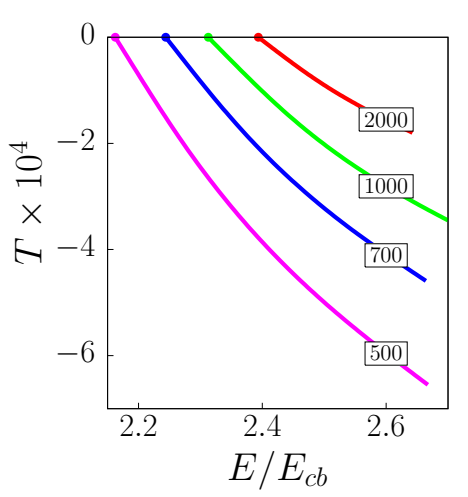

(b)

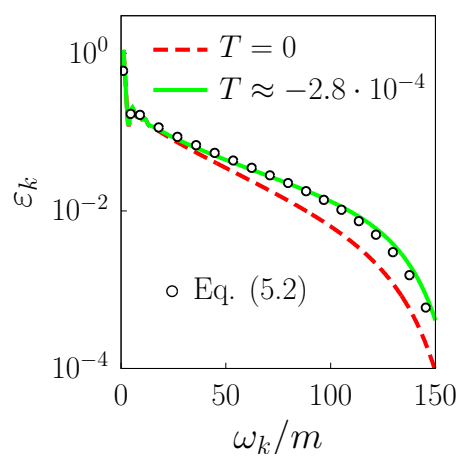

(c)

Figure 9. (a) Numerical solution $\phi_{s}(t, x)$ at $E \approx 2.6 E_{\mathrm{cb}}>E_{\mathrm{rt}}(N)$. The other parameters are $g^{2} N \approx 3.5, T \approx-2.8 \cdot 10^{-4}$, and $N_{x}=1000$. (b) Parameter $T$ in the region $E>E_{\mathrm{rt}}(N)$ for different $N_{x}$ (numbers in boxes). (c) Energy distributions for the solution (a) and the real-time instanton with $N_{x}=1000$ from figure 6b. In all figures $\theta=0.199$ and $N_{t}=11000$.

energy $E_{0}$ and one spectator particle carries the energy excess $\Delta E=E-E_{0}$, see figure 10 . The suppression exponent of the last process is $F_{N-1}\left(E_{0}\right) \approx F_{N}\left(E_{0}\right)$, where correction of order $g^{2} \theta \ll 1$ is ignored, cf. eq. (3.5). Since the inclusive transition is less suppressed than the exclusive one, we conclude that $F_{N}(E) \leq F_{N}\left(E_{0}\right)$, i.e. $F_{N}(E)$ is a non-increasing function of energy.

The above argument suggests that the negative values of $T \propto-\partial_{E} F_{N}$ in figure $9 \mathrm{~b}$ are lattice artifacts and one should be careful with the continuum limit $\Delta x \rightarrow 0$. Indeed, figure 9c shows that the high-frequency modes of solutions with $E>E_{\mathrm{rt}}(N)$ are enhanced as compared to the case of the real-time instanton and eq. (4.3). To perform the quantitative comparison, we boldly assume that the semiclassical solutions at $T<0$ have the form $\phi_{s}(x)=\phi_{\mathrm{rt}}(x)+\delta \phi(x)$, where $\delta \phi$ consists of high-frequency modes evolving linearly in the real-time instanton background. Ignoring reaction of the latter on $\delta \phi$, we write,

$$
\delta \phi(x)=\int \frac{d k}{4 \pi \omega_{k}}\left[\delta c_{k} \mathrm{e}^{-i k \cdot x}+\delta c_{k}^{*} \mathrm{e}^{i k \cdot x}\right] .
$$

This solution is real at $t \rightarrow+\infty$ because $\delta c_{k}$ and $\delta c_{k}^{*}$ are the mutually conjugate constants. 
One expresses $\delta c_{k}$ from the initial condition $(3.2 \mathrm{~d}): \delta c_{k}=\left(b_{k}^{(\mathrm{rt})} \gamma_{k}-a_{k}^{(\mathrm{rt})}\right) /\left(1-\gamma_{k}\right)$, where $\gamma_{k}=\mathrm{e}^{-2 \omega_{k} T-\theta}$. Here and below we mark quantities related to the real-time instanton with "(rt)", e.g. $a_{k}^{(\mathrm{rt})}=\mathrm{e}^{-\theta} b_{k}^{(\mathrm{rt})}$. We obtain the energy distribution for the solution with $T<0$,

$$
\varepsilon_{k} \equiv \frac{1}{4 \pi}\left(a_{k}^{(\mathrm{rt})}+\delta c_{k}\right)\left(b_{k}^{(r t) *}+\delta c_{k}^{*}\right)=\varepsilon_{k}^{(\mathrm{rt})} \frac{\sinh ^{2}(\theta / 2)}{\sinh ^{2}\left(\omega_{k} T+\theta / 2\right)},
$$

where $\varepsilon_{k}^{(\mathrm{rt})}=a_{k}^{(\mathrm{rt})} b_{k}^{(r t) *} / 4 \pi$. Recall that eq. (5.2) is based on a crude assumption that the solutions with $E>E_{\mathrm{rt}}(N)$ differ from the real-time instantons only in linearly evolving high-frequency modes. Nevertheless, this scaling works well: in figure $9 \mathrm{c}$ the function (5.2) (points) coincides with the actual energy distribution (solid line). In appendix B we derive eq. (5.2) in somewhat different approach.

Distributions (5.2) are qualitatively different at $T>0$ and $T<0$. In the former case the high-frequency asymptotics of $\varepsilon_{k}$ is consistent with eq. (4.3) which implies smooth continuum limit. At $T<0$, however, the function (5.2) develops a nonintegrable singularity at $\omega_{k}=\omega_{*} \equiv-\theta / 2 T$. Technically, this feature is related to the fact that the linear mode with frequency $\omega_{*}$ sat-

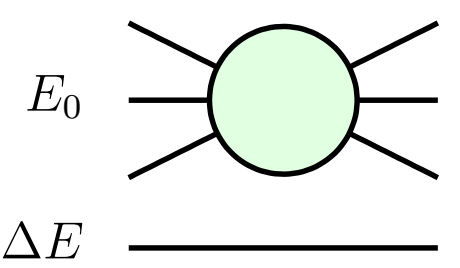
isfies reality conditions at $t \rightarrow \pm \infty$, see eqs. (3.2b) and (3.2d). Solutions can accumulate macroscopic energy in this mode before its amplitude $\delta c_{k_{*}} / \omega_{*} \sim \varepsilon_{k_{*}}^{1 / 2} / \omega_{*}$ becomes large and linear approximation (5.1) breaks down. Importantly, the latter energy tends to infinity as $T \rightarrow-0$ or $\omega_{*} \rightarrow+\infty$.

Lattice solutions do not feel the singularity in eq. (5.2) if the maximal lattice frequency $\omega_{\max }=2 / \Delta x$ is below $\omega_{*}$. If we decrease $\Delta x$ with constant $(T, \theta)$, the value of $\omega_{\max }$ approaches $\omega_{*}$ and the solution gains energy. Vice versa, at fixed energy one obtains smaller $|T|$ at smaller $\Delta x$. If eq. (5.2) is valid up to infinitesimally small $\Delta x$, parameter $T$ approaches zero as $\Delta x \rightarrow 0$, so that $\omega_{*}$ is kept outside of the lattice frequency range and the energy remains finite. The respective semiclassical solutions arrive at the realtime instanton plus modes with infinitely high frequency and vanishingly small amplitude carrying the energy excess $E-E_{\mathrm{rt}}(N)$. These solutions do not have smooth continuum limits.

The scaling property of our solutions is best summarized by dividing the total energy into "soft" and "hard" parts, i.e. $E=E_{\text {soft }}+E_{\text {hard }}$, involving modes with $\omega_{k}<\omega_{\Lambda}$ and $\omega_{\Lambda}<\omega_{k}<\omega_{\max }$ in eq. (3.3). In figure 11 we plot $E_{\text {soft }}(T)$ and $E_{\text {hard }}(T)$ for $\omega_{\Lambda}=70 m$ and several values of $N_{x}$. Predictably, $E_{\text {soft }}$ is not sensitive to $N_{x}$, while $E_{\text {hard }}$ sharply depends on it according to eq. (5.2) (lines in figure 11b). Since the data points in figure 11b are well approximated by eq. (5.2), the value of $T$ approaches zero as $N_{x} \rightarrow+\infty$ at fixed $E_{\text {hard }}$, so that $E_{\text {soft }} \rightarrow E_{\text {soft }}^{(\mathrm{rt})}$ in figure 11a.

A remark is in order. Our numerical results show that at high enough $E-E_{\mathrm{rt}}(N)$ the singularity $\omega_{*}$ falls below $\omega_{\max }$ and interaction of high-frequency modes becomes important. 


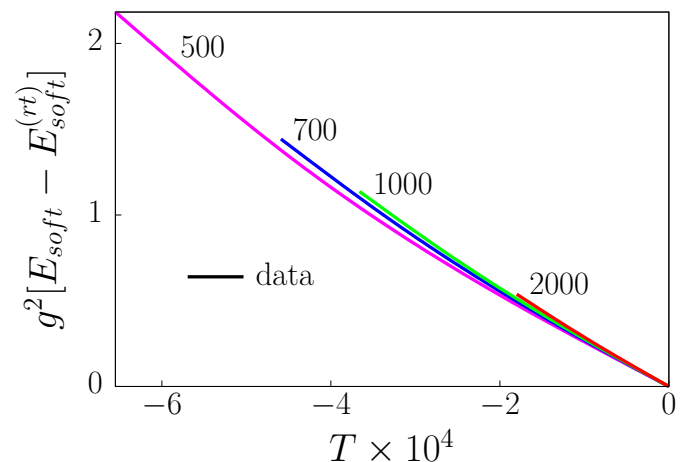

(a)

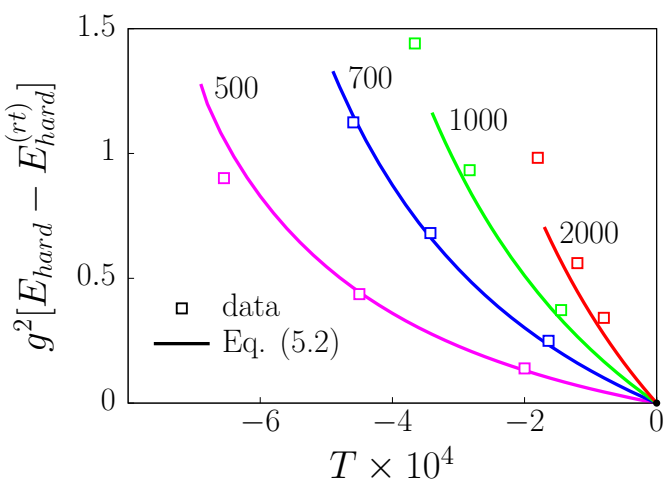

(b)

Figure 11. Energies of (a) soft and (b) hard modes of solutions at $E>E_{\mathrm{rt}}(N)$ and different $N_{x}$ (numbers near the lines); $\theta=0.199$.

We find, however, that the above qualitative picture holds even in this case. In particular, $|T|$ decreases with $N_{x}$ at fixed total energy $E$, see figure $9 \mathrm{~b}$. We therefore expect that the respective continuum limit has the same properties: parameter $T$ vanishes as $N_{x} \rightarrow \infty$ and lattice solutions approach the real-time instanton plus high-frequency modes.

It is important to point out that the lattice solutions lacking smooth continuum limits at $E>E_{\mathrm{rt}}(N)$, are still capable of describing tunneling transitions in that region. Namely, instead of discretizing the semiclassical equations (3.2) one can start from the lattice path integral for the cross section (3.1). The latter is an ordinary integral over $N_{t} \times 2 N_{x}$ variables $\phi_{i j}$. At $g^{2} \rightarrow 0$ it can be evaluated in the saddle-point approximation, where the saddlepoint values of $\phi_{i j}$ are the semiclassical lattice solutions. In this approach the existence of well-defined continuum limits of the latter solutions is irrelevant. The overall semiclassical results are reliable if the exponent $F_{N}(E)$ has a limit at $\Delta x \rightarrow 0$. Then the lattice solutions point at the dominant mechanism of quantum transition.

We have already argued that our lattice solutions with $E>E_{\mathrm{rt}}(N)$ describe processes shown in figure 10: they tend to the real-time instanton with energy $E_{\mathrm{rt}}(N)$ plus a few spectator particles in the form of high-frequency waves carrying the energy excess $E-$ $E_{\mathrm{rt}}(N)$. The respective suppression exponent is constant because $T \propto-\partial_{E} F_{N}$ approaches zero as $\Delta x \rightarrow 0$. We finally conclude that $F_{N}(E)$ is constant and equal to $F_{\min }(N)$ at energies above the threshold $E_{\mathrm{rt}}(N)$, see figure 1c and cf. refs. [24, 26].

\section{Stability of perturbative expansion around the real-time instanton}

Since the inclusive cross section does not grow exponentially with energy at $E>E_{\mathrm{rt}}$, one assumes that the terms of its perturbative expansion in $g^{2}$ around the real-time instanton are also bounded as $E \rightarrow+\infty$. Then one can compute the collision-induced amplitudes and cross sections perturbatively at $N=2$ and arbitrary high energies.

To test this property, we directly address the two-particle inclusive cross section of collision-induced false vacuum decay,

$$
\sigma(E)=\sum_{\Psi_{f}}\left|\left\langle\Psi_{f}\left|\hat{U}\left(t_{f}, t_{i}\right) \hat{a}_{p_{2}}^{\dagger} \hat{a}_{p_{1}}^{\dagger}\right| \Psi_{0}\right\rangle\right|^{2}
$$


where $t_{i, f} \rightarrow \mp \infty$, the initial state describes two particles with total momentum $P \equiv$ $p_{1}+p_{2}=(E, 0)$ in the false vacuum $\Psi_{0}$, each final state $\Psi_{f}$ contains an expanding bubble of true vacuum, and we ignore prefactors. Let us check the possibility of extracting the cross section 6.1 from the perturbative Green's functions in the backgrounds of the real-time instantons, so that the corrections to the perturbative calculations remain small as $E \rightarrow+\infty$.

In this section we compute only the "factorized" contribution to $\sigma(E)$ which was dominant and responsible for the exponential growth of the inclusive cross section in the Euclidean approach of section 2. We will see that this contribution is exponentially suppressed if the real-time instanton is used as a background. A consistent perturbative expansion around $\phi_{\mathrm{rt}}(x)$ will be developed in the next section.

We start from the Green's function (1.4) between the false vacuum $\Psi_{0}$ and the dominant final state $\Psi_{\mathrm{rt}}$ of the real-time instanton $\phi_{\mathrm{rt}}(x)$. In the leading order of the perturbation theory we substitute $\phi$ with $\phi_{\mathrm{rt}}\left(x-x_{0}\right)$ in the integrand of eq. (1.4) and find,

$$
\mathcal{G}_{\mathrm{rt}}=\mathcal{A}_{\mathrm{rt}} \int d^{2} x_{0} \Psi_{\mathrm{rt}}^{*}\left[\phi_{\mathrm{rt}}\right] \phi_{\mathrm{rt}}\left(x_{1}-x_{0}\right) \ldots \phi_{\mathrm{rt}}\left(x_{n+2}-x_{0}\right)
$$

where $^{19}$

$$
\mathcal{A}_{\mathrm{rt}}=\mathrm{e}^{i S\left[\phi_{\mathrm{rt}}\right]} \Psi_{0}\left[\phi_{\mathrm{rt}}\right] .
$$

Note that the position $x_{0}$ of $\phi_{\mathrm{rt}}$ is not fixed by the semiclassical equations (3.2). Nevertheless, the final-state wave functional $\Psi_{\mathrm{rt}}\left[\phi_{\mathrm{rt}}\right]$ depends on $x_{0}$ via $\phi_{\mathrm{rt}}\left(x-x_{0}\right)$.

We use the LSZ reduction formula and turn eq. (6.2) into the $2 \rightarrow n+\Psi_{\mathrm{rt}}$ amplitude. Considering the initial particles, we trade two of $\phi$ 's in the integrand for their positivefrequency residues $b_{p}^{*} \mathrm{e}^{-i p \cdot x_{0}}$, see eq. (3.2c). The case of the final-state particles is more involved because at $t \rightarrow+\infty$ the configuration $\phi_{\text {rt }}$ contains, apart from the outgoing waves, an interacting bubble. To handle this difficulty, we assume that the false vacuum decay occurs in a finite volume $|x|<L$ with periodic boundary conditions. Then at $t \rightarrow+\infty$ the bubble fills all space and $\phi_{\mathrm{rt}}(x)$ reduces to waves in the true vacuum,

$$
\phi_{\mathrm{rt}}\left(x-x_{0}\right) \rightarrow \phi_{+}+\int \frac{d k}{4 \pi \omega_{k}^{(+)}}\left[c_{k} \mathrm{e}^{-i k \cdot\left(x-x_{0}\right)}+c_{k}^{*} \mathrm{e}^{i k \cdot\left(x-x_{0}\right)}\right] \quad \text { as } \quad t \rightarrow+\infty,
$$

where $k^{\mu}=\left(\omega_{k}^{(+)}, k\right)$ are the on-shell momenta in the vacuum $\phi_{+}$. The LSZ formula substitutes $\phi_{\mathrm{rt}}\left(x-x_{0}\right)$ with its residue $c_{k} \mathrm{e}^{i k \cdot x_{0}}$ for every final-state particle. For clarity below we consider finite large $L$ and do not study the limit $L \rightarrow+\infty$.

We obtain,

$$
\mathcal{A}_{2 \rightarrow n+\Psi_{\mathrm{rt}}}^{\prime}=\mathcal{A}_{\mathrm{rt}} \int d^{2} x_{0} \frac{b_{p_{1}}^{*} b_{p_{2}}^{*}}{g^{2+n}} c_{k_{1}} \ldots c_{k_{n}} \Psi_{\mathrm{rt}}^{*}\left[\phi_{\mathrm{rt}}\right] \mathrm{e}^{i x_{0} \cdot\left(k_{1}+\cdots+k_{n}-p_{1}-p_{2}\right)} .
$$

Here $p_{j}$ and $k_{i}$ are the momenta of the initial and final particles, the prime of $\mathcal{A}^{\prime}$ reminds that only the factorized contribution is considered. Unlike in section 2, we do not explicitly

\footnotetext{
${ }^{19}$ Recall that $S$ and $\Psi_{0}$ are independent of $x_{0}$ in the limit $t_{i, f} \rightarrow \mp \infty$, as we drop the terms oscillating with $t_{i}$ and $t_{f}$.
} 
integrate over $x_{0}$ because $\Psi_{\mathrm{rt}}$ depends on it in a nontrivial way. Indeed, since $\phi_{\mathrm{rt}}(x)$ is real at $t \rightarrow+\infty$, its dominant final state is a coherent one $[46,63]$ : in the interaction representation

$$
\left|\Psi_{\mathrm{rt}}\right\rangle=\exp \left\{\int d k \frac{c_{k} \hat{c}_{k}^{\dagger}}{4 \pi g^{2} \omega_{k}^{(+)}}\right\}\left|\Psi_{+}\right\rangle
$$

where we introduced creation operators $\hat{c}_{k}^{\dagger}$ in the true vacuum $\Psi_{+}$. Parameters $c_{k}$ of $\Psi_{\mathrm{rt}}$ are precisely the final-state residues $c_{k}$ in eq. (6.4). One can extract dependence of the wave functional $\Psi_{\mathrm{rt}}\left[\phi_{\mathrm{rt}}\right]$ on $x_{0}$ from its transformation properties $\hat{c}_{k}^{\dagger} \rightarrow \hat{c}_{k}^{\dagger} \mathrm{e}^{-i k \cdot x_{0}}$ and $\Psi_{+} \rightarrow \mathrm{e}^{-i t_{0} E_{+}} \Psi_{+}$under spacetime shifts $x \rightarrow x+x_{0}$, where $E_{+}=2 L V\left(\phi_{+}\right)<0$ is the energy of the true vacuum.

The amplitude (6.5) has almost factorized form. In appendix A we integrate over the phase-space volume and obtain the inclusive cross section,

$$
\sigma^{\prime}(E)=\left|\mathcal{A}_{\mathrm{rt}} b_{p_{1}} b_{p_{2}} \Psi_{+}\left[\phi_{\mathrm{rt}}\right]\right|^{2} \int d^{2} \lambda \exp \left\{i P \cdot \lambda-i E_{+} \lambda^{0}+\int \frac{d k\left|c_{k}\right|^{2}}{4 \pi g^{2} \omega_{k}^{(+)}} \mathrm{e}^{-i k \cdot \lambda}\right\},
$$

where $P \equiv p_{1}+p_{2}=(E, 0)$. Equation (6.7) looks similar to eq. (2.7) of section 2, and yet it is entirely different. First, the initial-state factor $\left|b_{p_{1}} b_{p_{2}}\right|^{2} \propto \mathrm{e}^{-2 E T_{*}}$ decays exponentially with energy because higher momentum transfer from the initial particles to the soft background is less probable, see eq. (4.3). Second, the final-state contribution is nontrivial because $\Psi_{\mathrm{rt}}$ is not an eigenstate of energy, it absorbs different energies in different cases. Thus, the simple picture of converting all energy into the multiparticle final states with huge phase volume is lost.

Since the exponent in eq. (6.7) is large, the integral over $\lambda$ is evaluated in the saddlepoint approximation. One finds the extremum of the exponent $\lambda_{s}^{\mu}=\left(-2 i T^{\prime}, 0\right)$ satisfying

$$
E=E_{+}+\int d k \frac{\left|c_{k}\right|^{2}}{4 \pi g^{2}} \mathrm{e}^{-2 T^{\prime} \omega_{k}^{(+)}}
$$

At $E=E_{\mathrm{rt}}$ the solution is $T^{\prime}=0$ because the right-hand side of eq. (6.8) coincides with the final energy of the real-time instanton, cf. eq. (6.4). At $E>E_{\mathrm{rt}}$ one obtains $T^{\prime}<0$. Substituting $\lambda_{s}$ into eq. (6.8), we find,

$$
\sigma^{\prime}(E)=\left|\mathcal{A}_{\mathrm{rt}} \Psi_{+}\left[\phi_{\mathrm{rt}}\right]\right|^{2} \exp \left\{2 E\left(T^{\prime}-T_{*}\right)-2 E_{+} T^{\prime}+\int \frac{d k\left|c_{k}\right|^{2} \mathrm{e}^{-2 T^{\prime} \omega_{k}^{(+)}}}{4 \pi g^{2} \omega_{k}^{(+)}}\right\}=\mathrm{e}^{-F^{\prime}(E) / g^{2}}
$$

where the suppression exponent is introduced in the second equality.

Equation (6.8) implies that the suppression exponent of $\sigma^{\prime}(E)$ grows at $E>E_{\mathrm{rt}}$,

$$
\frac{d F^{\prime}}{d E}=2 g^{2}\left(T_{*}-T^{\prime}\right)>0
$$

In the next section we will demonstrate that at $E=E_{\mathrm{rt}}$ the exponent $F^{\prime}(E)$ coincides with the true semiclassical exponent $F(E)$. The latter, however, is constant at high energies. Thus, the factorized contribution (6.9) is exponentially subdominant, $F^{\prime}>F$, at $E>E_{\mathrm{rt}}$. 


\section{$7 \quad$ Perturbative method at high energies}

Before considering the dominant contribution, we remark that the perturbative approach of the previous section is incomplete. Indeed, there is a large family of real-time instantons parametrized $^{20}$ with $N=N_{0}$ and position $y_{0}$. Every solution from this family has its own dominant final state $\Psi_{\mathrm{rt}}$ which also depends on $N_{0}$ and $y_{0}$, cf. eq. (6.6). In this section we change notation $N \rightarrow N_{0}$ for the real-time instanton parameter, to distinguish it from the number of colliding particles in the process; from now on, the latter equals two. We stress that the values of $N_{0}$ and $y_{0}$ characterize the background for the perturbative expansion. Their values are selected to achieve better convergence.

Our receipt for perturbative evaluation of the collision-induced cross section at high energies and two initial particles is summarized as follows [29]. One starts from the $(n+2)$ point Green's function (1.4) between the false vacuum $\Psi_{0}$ and the dominant final state $\Psi_{\mathrm{rt}}$ of the real-time instanton $\phi_{\mathrm{rt}}^{\left(N_{0}\right)}\left(x-y_{0}\right)$, cf. eq. (6.6). One evaluates the path integral for the Green's function perturbatively in the background of $\phi_{\mathrm{rt}}$ : substitutes $\phi(x)=\phi_{\mathrm{rt}}^{\left(N_{0}\right)}(x-$ $\left.x_{0}\right)+g \delta \phi(x)$ and expands the integrand in $g \delta \phi$. Integral over the would-be flat direction $x_{0} \neq y_{0}$ remains in the Green's function, cf. eq. (6.2). One finally extracts the perturbative amplitudes $\mathcal{A}_{2 \rightarrow n+\Psi_{\mathrm{rt}}}$ from the LSZ formula, turns them into the inclusive cross section with the machinery of appendix A, and takes the limit $N_{0} \rightarrow 0$. The result is a perturbative expansion for $\sigma(E)$ which, as we are going to argue, is applicable at arbitrary high energies.

Let us explain the role of the auxiliary parameter $N_{0}$ characterizing the background solution. On the one hand, recall that the real-time instanton $\phi_{\mathrm{rt}}^{\left(N_{0}\right)}$ describes transition from the initial states with $N_{0}$ particles: the limit $N \equiv N_{0} \rightarrow 0$ formally corresponds to the vacuum initial state. Indeed, at $\theta \rightarrow+\infty$ one simultaneously obtains Feynman initial condition $a_{k} \rightarrow 0$ for $\phi_{\mathrm{rt}}^{\left(N_{0}\right)}$ and $N_{0} \rightarrow 0$, see eqs. (3.2d) and (3.3). Thus, the real-time instanton with $N_{0}=0$ and $x_{0}=y_{0}$ is the formal saddle-point configuration ${ }^{21}$ for the path integral (1.4), and the perturbative series around it constitute the ordinary saddle-point expansion. On the other hand, the energy $E_{\mathrm{rt}}\left(N_{0}\right)$ of the real-time instanton represents the extremum, $\partial_{E} F_{N}=0$, and stays nonzero in the limit $N_{0} \rightarrow 0$, cf. figure $8 \mathrm{~b}$. This means that $\phi_{\mathrm{rt}}^{\left(N_{0}\right)}$ is singular at $N_{0}=0$ because its typical frequencies $\omega_{k} \sim E / N_{0}$ are infinite. We therefore develop perturbative expansion around the smooth configurations with $N_{0}>0$ and send $N_{0} \rightarrow 0$ in the end of calculations.

In figure 12 we plot parameter $\mathrm{e}^{-\theta}$ of the real-time instanton and distance $T_{*}$ to its closest singularity as functions of $N_{0}$. Numerical data (solid lines) are well fitted by quadratic polynomials with zeros at $N_{0}=0$ (dashed lines). The graphs support our expectation that as $N_{0} \rightarrow 0$, the real-time instanton $\phi_{\mathrm{rt}}^{\left(N_{0}\right)}$ tends to a singular configuration with vacuum initial conditions.

\footnotetext{
${ }^{20}$ Recall that we work in the finite- $L$ box which explicitly breaks Lorentz symmetry. Boosted real-time instantons should be taken into account in the infinite-volume limit.

${ }^{21}$ Recall that $\phi_{\mathrm{rt}}$ extremizes the classical action and, by construction of $\Psi_{\mathrm{rt}}$, at $x_{0}=y_{0}$ serves as the saddle-point configuration for the integral with the final state in eq. (1.4). Note also that the two initial particles of the process are represented by the two $\phi$-factors in the integrand which do not change the initial saddle-point conditions $a_{k}=0$. The latter are satisfied by $\phi_{\mathrm{rt}}^{\left(N_{0}\right)}$ at $N_{0} \rightarrow 0$.
} 


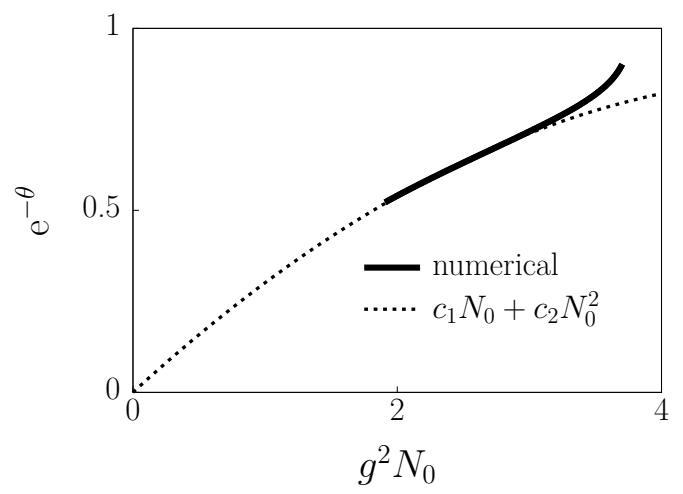

(a)

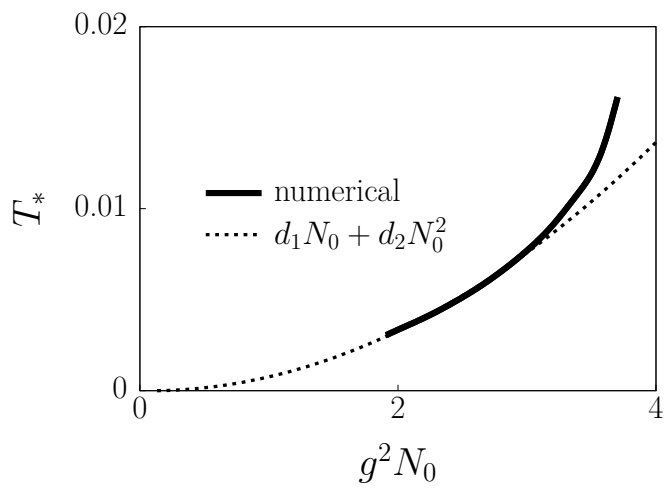

(b)

Figure 12. Quantities (a) $\mathrm{e}^{-\theta}$ and (b) $T_{*}$ characterizing the real-time instanton as functions of its parameter $N_{0}$. Numerical data at $N_{t} \times N_{x}=11000 \times 3000$ (solid lines) are fitted with quadratic polynomials in $N_{0}$ (dotted lines).

Expanding the integrand of eq. (1.4) in $g \delta \phi$, we obtain Feynman rules involving points, propagators and vertices,

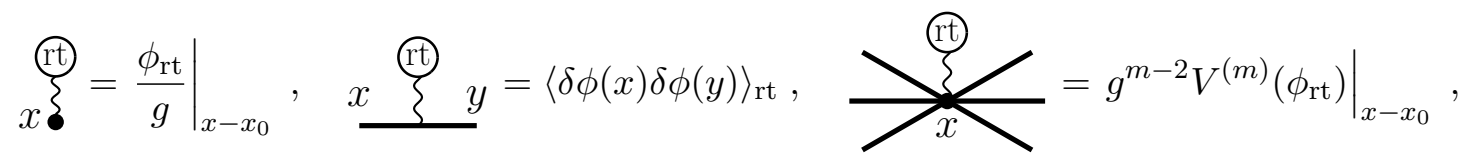

which explicitly depend on $N_{0}$ and $x_{0}$. At nonzero $N_{0}$ or $x_{0} \neq y_{0}$ one also obtains the tadpoles, i.e. terms in action proportional to $\delta \phi$, which characterize deviation of the background solution from the true saddle-point configuration. The tadpoles coming from the terms at $t \rightarrow-\infty$ vanish as $N_{0} \rightarrow 0$, and we do not consider them in what follows. The final-state tadpoles are related to the fact that $\Psi_{\mathrm{rt}}$ is the dominant final state for the configuration $\phi_{\mathrm{rt}}\left(x-y_{0}\right)$ which is different from our background $\phi_{\mathrm{rt}}\left(x-x_{0}\right)$. We will discuss them in the end of this section. Since the elements in eq. (7.1) explicitly depend on $x$, energy and momentum are not conserved along the lines and in the vertices. Rather, the Feynman rules in momentum space involve structure functions depending on the momentum $Q$ transferred to the background. We will see shortly that the real-time instanton consumes total energy $Q^{0} \approx E_{\mathrm{rt}}$ from the initial particles.

At zeroth order of the perturbative expansion one uses $\phi(x)=\phi_{\mathrm{rt}}^{\left(N_{0}\right)}\left(x-x_{0}\right)$ in eq. (1.4) and obtains the diagram in figure 13a. Extracting the cross section and summing over the final states, one arrives at the contribution (6.9) which is exponentially subdominant. Indeed, we argued in eq. (6.10) that the suppression exponent $F^{\prime}(E)$ of this contribution grows with energy at $E>E_{\mathrm{rt}}$. Besides, at $E=E_{\mathrm{rt}}$ and $N_{0} \rightarrow 0$ we have $T^{\prime}=T_{*}=0$ and therefore

$$
F^{\prime}\left(E_{\mathrm{rt}}\right)=2 g^{2} \operatorname{Im} S\left[\phi_{\mathrm{rt}}\right]-g^{2} \ln \left|\Psi_{0}\right|^{2}-g^{2} \ln \left|\Psi_{+}\right|^{2}-\int \frac{d k\left|c_{k}\right|^{2}}{4 \pi \omega_{k}^{(+)}}=F\left(E_{\mathrm{rt}}\right)+O\left(N_{0}\right)
$$




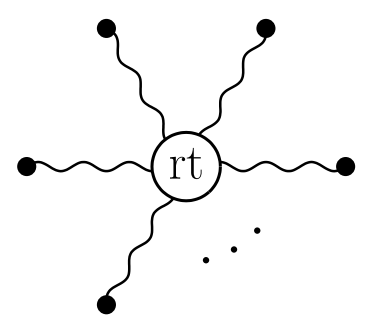

(a)

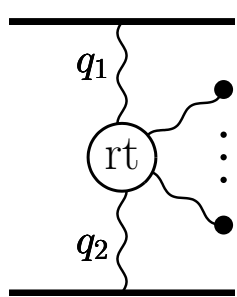

(b)

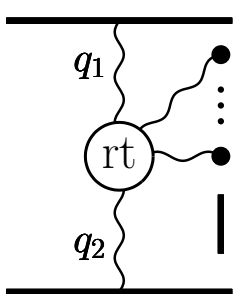

(c)

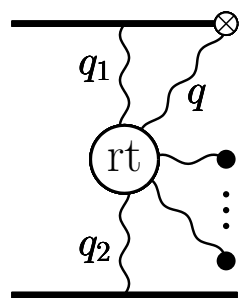

(d)

Figure 13. Diagrams for perturbative expansion around the real-time instanton.

Here we used eqs. (6.3) and (3.4), vacuum wave functionals

$$
\Psi_{0}[\phi]=\exp \left\{-\int \frac{d k \omega_{k}}{4 \pi g^{2}} \phi(k) \phi(-k)\right\}_{t_{i}}
$$

and $\Psi_{+}[\phi]$, spatial Fourier transform $\phi(k)$ of configuration $\phi$, and representations (3.2c), (6.4) of the real-time instanton. ${ }^{22}$ Since the dominant exponent $F(E)$ is constant at high energies, we repeat that $F^{\prime}(E)>F(E)$ at $E>E_{\text {rt }}$, i.e. the factorized diagram in figure 13a is negligible. ${ }^{23}$

The dominant diagram at $E>E_{\mathrm{rt}}$ is shown in figure 13b. It describes propagation of two initial particles which transfer momentum $Q=q_{1}+q_{2}$ to the background. The respective transition amplitude is, ${ }^{24}$

$$
\mathcal{A}_{2 \rightarrow n+\Psi_{\mathrm{rt}}}=\mathcal{A}_{\mathrm{rt}} n(n-1) \int d^{2} x_{0} D_{p_{1}, k_{1}} D_{p_{2}, k_{2}} \frac{c_{k_{3}} \ldots c_{k_{n}}}{g^{n-2}} \Psi_{\mathrm{rt}}^{*}\left[\phi_{\mathrm{rt}}\right] \mathrm{e}^{i x_{0} \cdot\left(k_{3}+\cdots+k_{n}-q_{1}-q_{2}\right)},
$$

cf. eq. (6.5). Here $q_{i} \equiv p_{i}-k_{i}$ and $D_{p, k} \cdot \mathrm{e}^{-i q \cdot x_{0}}$ is the double residue of the propagator $\langle\delta \phi \delta \phi\rangle_{\mathrm{rt}}$. In appendix A we convert the amplitude into the inclusive cross section,

$$
\begin{aligned}
\sigma(E)= & \left|\mathcal{A}_{\mathrm{rt}} \Psi_{+}\left[\phi_{\mathrm{rt}}\right]\right|^{2} \int \frac{d k_{1} d k_{2}}{32 \pi^{2} \omega_{k_{1}}^{(+)} \omega_{k_{2}}^{(+)}}\left|D_{p_{1}, k_{1}} D_{p_{2}, k_{2}}+D_{p_{1}, k_{2}} D_{p_{2}, k_{1}}\right|^{2} \\
& \times \int d^{2} \lambda \exp \left\{i Q \cdot \lambda-i E_{+} \lambda^{0}+\int \frac{d k\left|c_{k}\right|^{2}}{4 \pi g^{2} \omega_{k}^{(+)}} \mathrm{e}^{-i k \cdot \lambda}\right\},
\end{aligned}
$$

where $\lambda \equiv y_{0}-x_{0}$ and we omit trivial prefactors. The first line in this expression is the naive Feynman diagram in the background of the real-time instanton. The factor in the second line is related to the on-shell final state of the background process. It is the same as in eq. (6.7). In section 6 we demonstrated that this factor is sharply peaked around $Q^{\mu}=\left(E_{\mathrm{rt}}, 0\right)$ with fluctuations of order $\Delta Q \sim g E_{\mathrm{rt}}$.

\footnotetext{
${ }^{22}$ Recall also that $F_{N}(E)=F(E)-g^{2} \theta N+O(N)$ as we argued in the end of section 3 .

${ }^{23}$ One finds that as $L \rightarrow \infty$, the energy of the true vacuum becomes infinite and therefore $T^{\prime} \rightarrow 0$. This means that in the true infinite-volume limit (not considered here) the contribution (6.9) becomes comparable to the dominant one.

${ }^{24}$ Symmetrization with respect to permutations of $k_{1}, \ldots, k_{n}$ is assumed.
} 
Evaluating the integrals over $Q$ and $\lambda$ in the saddle-point approximation, we get with exponential precision,

$$
\sigma(E)=\mathrm{e}^{-F\left(E_{\mathrm{rt}}\right) / g^{2}} \quad \text { at } \quad E>E_{\mathrm{rt}},
$$

see eqs. (6.9) and (7.2). We thus obtained constant suppression exponent $F=F\left(E_{\mathrm{rt}}\right)$ at $E>E_{\mathrm{rt}}$ which was deduced in section 5 on the basis of sophisticated numerical analysis. Note that our perturbative method can be applied for calculating the prefactor: one just has to estimate the energy-independent saddle-point determinant in the Green's function (1.4) and collect few simple prefactors in the above calculations.

The first perturbative correction to the dominant contribution is shown in figure 13c. It involves the same relative factor $g^{2} n^{2}$ as in the low-energy calculation of section 2. This time, however, the number $n$ of the point-like factors $\phi_{\mathrm{rt}}$ representing the particles added to the background, is relatively small and does not grow with the collision energy $E$. Indeed, any energy transfer to the background above $Q=E_{\mathrm{rt}}$ is cut off by the final-state factor. This means that $n \sim\left|Q-E_{\mathrm{rt}}\right| / m \sim g^{-1}$, and the diagram in figure $13 \mathrm{c}$ is of the same order as the dominant one. Importantly, it does not grow with energy. Resummation of these disconnected diagrams $[51,52]$ leaves us with corrections involving vertices which are suppressed by the true expansion parameter $g$.

Let us finally discuss the tadpoles. We saw that the parameter $y_{0}$ of $\Psi_{\mathrm{rt}}$ does not coincide with the position $x_{0}$ of the background solution: the integral over the difference $\lambda \equiv y_{0}-x_{0}$ enters eq. (7.4). Thus, $\phi_{\mathrm{rt}}\left(x-x_{0}\right)$ is not the true saddle-point configuration of the integral (1.4), perturbative expansion around it starts from the linear term in $\delta \phi$. We obtain the tadpole

$$
\stackrel{\text { 까 }}{q}=\frac{c_{q}^{*} \mathrm{e}^{-i q \cdot x_{0}}}{4 \pi g \omega_{k}^{(+)}}\left(\mathrm{e}^{-i q \cdot \lambda}-1\right)
$$

which should be integrated over $q$ with the final-state residue of the propagator. Apart from the additional integration, this tadpole is similar to the final on-shell particle in the amplitude. One may think that the dominant contribution includes diagrams with the tadpoles attached to the hard propagators, e.g. figure 13d. However, all momentum $p_{1}=q_{1}+q$ of the propagator with the tadpole is transferred to the real-time instanton. Indeed, eq. (7.5) is proportional to the exponent $e^{-i q \cdot x_{0}}$ which accumulates transferred momentum in the amplitude, cf. eq. (7.3). Then the total transferred energy in the process in figure $13 \mathrm{~d}$ is higher than $E_{\mathrm{rt}}$ and the respective contribution to the cross section is exponentially small.

We see that the tadpoles infest the final-state propagators in figure $13 \mathrm{c}$ and subdominant diagrams. ${ }^{25}$ Note, however, that the element $(7.5)$ is $O\left(g^{0}\right)$ at best. Indeed, at small $q$ the bracket in eq. (7.5) is proportional to $g$ because $\lambda \sim g$ at $E \approx E_{\mathrm{rt}}$. At $q \sim m / g$ the tadpole is suppressed by the factor $c_{q}^{*} / \omega_{q}^{(+)}$which vanishes at high $q$ because the energy of the real-time instanton is finite. Thus, the tadpoles also do not break the perturbative expansion.

\footnotetext{
${ }^{25}$ One can check that the tedpoles do not change our conclusion about exponential suppression of the factorized contribution.
} 
We conclude that the perturbative expansion around the real-time instanton is reliable at arbitrarily high energies.

\section{Summary and discussion}

In this paper we studied collision-induced tunneling in field theory. We paid special attention to the case of two colliding particles with high total energy $E$. As a playground we considered induced false vacuum decay in $(1+1)$ dimensions. We demonstrated that the suppression exponent $F_{N}(E)$ of this process decreases with energy, reaches minimum $F=F_{\min }(N)$ at $E=E_{\mathrm{rt}}(N)$ and remains constant at higher energies.

Our methods rely on existence of the real-time instantons - a special class of semiclassical solutions describing inclusive collision-induced transitions from the initial states with $N$ particles and arbitrary energies. The minimal suppression $F_{\min }(N)$ and threshold energy $E_{\mathrm{rt}}(N)$ are computed as functionals on these solutions. Real-time instantons were first observed in the toy model of ref. [26]. Here we numerically obtained them in the case of $(1+1)$-dimensional false vacuum decay. We expect that these solutions exist for other collision-induced tunneling processes. One can verify this expectation on a case-to-case basis by solving the respective semiclassical boundary value problem in a given model.

Importantly, we argue on general grounds that the real-time instantons are complex solutions evolving in real time (hence the title). This property is very unusual for the semiclassical solutions related to exponentially suppressed transitions, it leads to far-reaching consequences. We find that scattering of high-energy quantum particles in the backgrounds of the real-time instantons resembles scattering in vacuum because energy exchange between the particles and the soft background occurs with exponentially small probability. This situation is radically different from that at low energies where the Euclidean semiclassical solutions recycle any additional energy into exponentially large probability factors. Starting from the real-time instantons, we develop a perturbative description of the twoparticle collision-induced processes at high energies. We demonstrate that this description remains valid at arbitrary high energies. Our method shows that the suppression exponent $F(E) \equiv F_{2}(E)$ is constant at $E>E_{\mathrm{rt}}(2)$. The collision-induced transitions in this regime involve transfer fixed energy $E_{\mathrm{rt}}$ from the two colliding particles to the soft background; the energy excess $E-E_{\mathrm{rt}}$ remains in the initial particles till the end of the process. Note that our perturbative methods can be easily generalized for calculating prefactors or exclusive cross sections.

We conclude that the real-time instantons, if exist for a given collision-induced process, provide powerful perturbative framework and guarantee constant suppression exponent $F(E)=F_{\min }(2)$ of this process at energies above a certain threshold $E_{\mathrm{rt}}(2)$.

\section{Acknowledgments}

We are grateful to S.M. Sibiryakov and F.L. Bezrukov for motivation and to V.A. Rubakov [64] for criticism. This work is supported by the RSCF grant 14-22-00161. D.L. thanks EPFL for hospitality. 


\section{A Multiparticle cross sections}

In this appendix we evaluate inclusive cross sections for the amplitudes considered in the main body of the paper. We start from the factorized $2 \rightarrow n$ amplitude

$$
\mathcal{A}_{2 \rightarrow n}=\frac{\mathcal{A}_{0}}{g^{n}} c_{k_{1}} \ldots c_{k_{n}} .
$$

Here $\mathcal{A}_{0}$ and $g$ are constants, $k_{i}$ are momenta of particles in the final state. The cross section of inclusive transition to the $n$-particle final states is obtained by integrating over the phase space volume $\Pi_{n}$,

$$
\begin{aligned}
\sigma_{n}(P) & =\int\left|\mathcal{A}_{2 \rightarrow n}\right|^{2} d \Pi_{n}(P) \\
& =\frac{\left|\mathcal{A}_{0}\right|^{2}}{n !} \int \frac{d k_{1}\left|c_{k_{1}}\right|^{2}}{4 \pi g^{2} \omega_{k_{1}}} \ldots \int \frac{d k_{n}\left|c_{k_{n}}\right|^{2}}{4 \pi g^{2} \omega_{k_{n}}}(2 \pi)^{2} \delta^{(2)}\left(k_{1}+\cdots+k_{n}-P\right),
\end{aligned}
$$

where we ignored the initial-state factor, introduced the total initial momentum $P_{\mu}$ and on-shell frequencies $\omega_{k}^{2}=k^{2}+m^{2}$. We use Fourier representation of the $\delta$-function and find,

$$
\sigma_{n}(P)=\frac{\left|\mathcal{A}_{0}\right|^{2}}{n !} \int d^{2} \lambda[f(\lambda)]^{n} \mathrm{e}^{i P \cdot \lambda}, \quad \text { where } \quad f(\lambda)=\int \frac{d k\left|c_{k}\right|^{2}}{4 \pi g^{2} \omega_{k}} \mathrm{e}^{-i k \cdot \lambda} .
$$

If $c_{k}=c_{\mathrm{b}}$ does not depend on $k$,

$$
f(\lambda)=\frac{\left|c_{b}\right|^{2}}{2 \pi g^{2}} K_{0}\left(m \sqrt{-\lambda^{2}+i \epsilon \lambda^{0}}\right) .
$$

Equation (A.2) shows that $\lambda$ is a typical Compton wavelength of the final particles, $\lambda \sim k^{-1}$. Summing up the $n$-particle contributions (A.2), we obtain eq. (2.7) of section 2.

In section 6 we consider the factorized amplitude (6.5) of the process $2 \rightarrow n+\Psi_{\mathrm{rt}}$. To simplify summation over the final states in the inclusive cross section, we relate eq. (6.5) to the ordinary $2 \rightarrow n+m$ amplitudes. To this end we expand the exponent in the final state (6.6) of our amplitude and obtain,

$$
\mathcal{A}_{2 \rightarrow n+\Psi_{\mathrm{rt}}}^{\prime}=\sum_{m=0}^{\infty} \frac{1}{m !} \int \frac{d k_{n+1} c_{k_{n+1}}^{*}}{4 \pi g \omega_{k_{n+1}}^{(+)}} \ldots \int \frac{d k_{n+m} c_{k_{n+m}}^{*}}{4 \pi g \omega_{k_{n+m}}^{(+)}} \mathcal{A}_{2 \rightarrow n+m}^{\prime}(2 \pi)^{2} \delta^{(2)}\left(P-P_{f}\right),
$$

where $P_{f}$ is the total momentum of the $(n+m)$-particle final state. Next, we compare the right-hand side of eq. (6.5) with eq. (A.3). Substituting $\phi=\phi_{\text {rt }}$ into the wave functional $\Psi_{d}[\phi]$ of a coherent state $[28]$ with parameters $d_{k} \mathrm{e}^{-i \omega_{k} t_{f}}$, we find,

$$
\Psi_{d}\left[\phi_{\mathrm{rt}}\right] \equiv\left\langle\phi \mid \Psi_{d}\right\rangle=\exp \left\{\int d k \frac{d_{k} c_{k}^{*} \mathrm{e}^{-i k \cdot x_{0}}}{4 \pi g^{2} \omega_{k}^{(+)}}\right\} \mathrm{e}^{-i E_{+} t_{0}} \Psi_{+}\left[\phi_{\mathrm{rt}}\right],
$$

where we extracted dependence on $x_{0}$ as explained in section 6, used the form (6.4) of $\phi_{\mathrm{rt}}$, took the limit $t_{f} \rightarrow+\infty \cdot \mathrm{e}^{-i \epsilon}$ and introduced wave functional $\Psi_{+}$of the true vacuum. The 
final state $\Psi_{\mathrm{rt}}$ in section 6 has $d_{k}=c_{k}$, cf. eq. (6.6). Expanding the exponent in eq. (A.4) and substituting the series into eq. (6.5), we find,

$$
\begin{aligned}
\mathcal{A}_{2 \rightarrow n+\Psi_{\mathrm{rt}}}^{\prime}= & \sum_{m=0}^{\infty} \frac{1}{m !} \int \frac{d k_{n+1}\left|c_{k_{n+1}}\right|^{2}}{4 \pi g^{2} \omega_{k_{n+1}}^{(+)}} \cdots \int \frac{d k_{n+m}\left|c_{k_{n+m}}\right|^{2}}{4 \pi g^{2} \omega_{k_{n+m}}^{(+)}} \\
& \times \frac{\mathcal{A}_{0}}{g^{n}} c_{k_{1}} \ldots c_{k_{n}}(2 \pi)^{2} \delta^{(2)}\left(P-P_{f}\right) .
\end{aligned}
$$

Here we evaluated the integral over $x_{0}$ and introduced $\mathcal{A}_{0}=\mathcal{A}_{\mathrm{rt}} \Psi_{+}^{*}\left[\phi_{\mathrm{rt}}\right] b_{p_{1}}^{*} b_{p_{2}}^{*} / g^{2}$. Comparing eqs. (A.3) and (A.5), one finds that the amplitudes $\mathcal{A}_{2 \rightarrow n+m}^{\prime}$ have the form (A.1). We finally obtain the inclusive cross section (6.7) by summing up the $n$-particle ones in eq. (A.2).

To process the dominant amplitude (7.3) of section 7, we do the opposite to the above, i.e. combine the final states into the coherent states $\Psi_{d}$ with parameters $d_{k} \equiv c_{k} \mathrm{e}^{i k \cdot y_{0}}+\delta c_{k}$, where $c_{k} \mathrm{e}^{i k \cdot y_{0}}$ are the parameters of $\Psi_{\mathrm{rt}}$ and $\delta c_{k}$ are arbitrary. We find,

$$
\begin{aligned}
\mathcal{A}_{2 \rightarrow \Psi_{d}} & =\sum_{n=0}^{\infty} \frac{1}{n !} \int \frac{d k_{1} \delta c_{k_{1}}^{*}}{4 \pi g \omega_{k_{1}}^{(+)}} \cdots \int \frac{d k_{n} \delta c_{k_{n}}^{*}}{4 \pi g \omega_{k_{n}}^{(+)}} \mathcal{A}_{2 \rightarrow n+\Psi_{f}^{r t}} \\
& =\mathcal{A}_{\mathrm{rt}} \int d^{2} x_{0} \Psi_{d}^{*}\left[\phi_{\mathrm{rt}}\right] \int \frac{d k_{1} d k_{2} \delta c_{k_{1}}^{*} \delta c_{k_{2}}^{*}}{16 \pi^{2} g^{2} \omega_{k_{1}}^{(+)} \omega_{k_{2}}^{(+)}} D_{p_{1}, k_{1}} D_{p_{2}, k_{2}} \mathrm{e}^{-i x_{0} \cdot\left(q_{1}+q_{2}\right)}
\end{aligned}
$$

In the first line of this expression we expanded $\Psi_{d}$ in $\delta c$ using eq. (6.6), in the second substituted eq. (7.3) and introduced wave functional $\Psi_{d}\left[\phi_{\mathrm{rt}}\right]$, eq. (A.4).

Now, we evaluate the inclusive cross section

$$
\sigma(E)=\frac{1}{V^{(2)}} \int \mathcal{D} d^{\prime} \mathcal{D} d^{*} \mathcal{A}_{2 \rightarrow \Psi_{d}} \mathcal{A}_{2 \rightarrow \Psi_{d^{\prime}}}^{*} \exp \left\{-\int \frac{d k d_{k}^{\prime} d_{k}^{*}}{4 \pi g^{2} \omega_{k}^{(+)}}\right\},
$$

where $V^{(2)}$ is the spacetime volume. First, we note that the amplitude (A.7) depends on the arbitrary parameter $y_{0}$. This is the freedom of choosing the background for expansion, it disappears after resummation of perturbative series. We fix the freedom requiring

$$
y_{0}=x_{0}^{\prime}, \quad y_{0}^{\prime}=x_{0}
$$

where $y_{0}^{\prime}$ and $x_{0}^{\prime}$ come from the complex conjugate amplitude (A.6) in the integral for the inclusive cross section. Second, the parameters $\delta c_{k}^{*}$ in the prefactor can be obtained by varying the exponent $\exp \left\{\int d k \alpha_{k} \delta c_{k}^{*} /\left(4 \pi g^{2} \omega_{k}^{(+)}\right)\right\}$with respect to $\alpha$ at $\alpha=0$. The integral in eq. (A.7) therefore can be evaluated using the functional

$$
\begin{aligned}
\Pi\left[\alpha, \alpha^{\prime *}\right] & =\int \mathcal{D} \delta c^{\prime} \mathcal{D} \delta c^{*} \Psi_{d}^{*}\left[\phi_{\mathrm{rt}}\right] \Psi_{d^{\prime}}\left[\phi_{\mathrm{rt}}\right] \exp \left[\int \frac{d k}{4 \pi g^{2} \omega_{k}^{(+)}}\left(\alpha_{k} \delta c_{k}^{*}+\alpha_{k}^{*} \delta c_{k}^{\prime}-d_{k}^{\prime} d_{k}^{*}\right)\right] \\
& =\mathrm{e}^{-i E_{+} \lambda^{0}}\left|\Psi_{+}\left[\phi_{\mathrm{rt}}\right]\right|^{2} \exp \left[\int \frac{d k}{4 \pi g^{2} \omega_{k}^{(+)}}\left(\alpha_{k} \alpha_{k}^{\prime *}+\left|c_{k}\right|^{2} \mathrm{e}^{-i k \cdot \lambda}\right)\right],
\end{aligned}
$$


where we denoted $d_{k}^{\prime} \equiv c_{k} \mathrm{e}^{i k \cdot y_{0}^{\prime}}+\delta c_{k}^{\prime}$, substituted eq. (A.4), evaluated the integrals over $\delta c^{*}, \delta c^{\prime}$ and denoted $\lambda \equiv y_{0}-x_{0}=x_{0}^{\prime}-x_{0}$. We see that variations over $\alpha_{k}$ and $\alpha_{k}^{\prime *}$ give contraction rule for the final-state variables $\delta c_{k_{1}} \delta c_{k_{2}}^{*}=4 \pi g^{2} \omega_{k}^{(+)} \delta\left(k_{1}-k_{2}\right)$.

Substituting eqs. (A.6) and (A.9) into eq. (A.7), one obtains the inclusive cross section (7.4) containing the integral over $\lambda=x_{0}^{\prime}-x_{0}$; integration with respect to $\left(x_{0}+x_{0}^{\prime}\right) / 2$ gives the two-volume $V^{(2)}$ which is canceled in eq. (A.7).

\section{B High-frequency tail of the semiclassical solution}

Let us evaluate high-frequency asymptotics of the saddle-point solution $\phi_{s}(x)$. To this end we represent $\phi_{s}(x)=\phi_{0}(x)+\delta \phi(x)$ as a sum of soft nonlinear background $\phi_{0}$ and highfrequency part $\delta \phi \ll \phi_{0}$ evolving on top of it. Functions $\phi_{0}$ and $\delta \phi$ contain modes with $k<\Lambda$ and $k>\Lambda$, respectively. One rewrites the field equation as

$$
\square \delta \phi(x)=J(x),
$$

where $J$ is a contribution of $\phi_{0}$ and $\delta \phi$ is ignored in potential terms. We solve eq. (B.1),

$$
\delta \phi(x)=-\int \frac{d^{2} k}{(2 \pi)^{2}} \frac{J(k) \mathrm{e}^{-i k \cdot x}}{k^{2}-i \epsilon k^{0}}+\int_{k>\Lambda} \frac{d k}{4 \pi \omega_{k}}\left[c_{k} \mathrm{e}^{-i k \cdot x}+c_{k}^{*} \mathrm{e}^{i k \cdot x}\right],
$$

using the two-dimensional Fourier image of the source $J(k)$ and arbitrary on-shell waves in the second term with $k^{\mu}=\left(\omega_{k}, k\right)$ and $\omega_{k}=|k|$. The solution (B.2) is real as $t \rightarrow+\infty$ in accordance with eq. (3.2b). In the infinite past, i.e. $t \rightarrow i T-\infty$, eq. (B.2) takes the form (3.2c) with

$$
a_{k}=c_{k}+J_{k}^{-}, \quad b_{k}^{*}=c_{k}^{*}+J_{k}^{+}, \quad \text { and } \quad J_{k}^{ \pm} \equiv \pm\left. i J(\mp k)\right|_{k^{0}=\omega_{k}} .
$$

We finally solve the initial condition (3.2d) and obtain,

$$
c_{k}=\frac{\gamma_{k}\left(J_{k}^{+}\right)^{*}-J_{k}^{-}}{1-\gamma_{k}}, \quad \gamma_{k} \equiv \mathrm{e}^{-2 \omega_{k} T-\theta}
$$

Note that eq. (B.1) and its solution (B.2), (B.4) are valid only at $k>\Lambda \gg m$.

One notices that the sources $J_{k}^{ \pm}$are exponentially sensitive to $k$. Indeed, the $t$-contours in the Fourier transforms

$$
J_{k}^{ \pm}= \pm\left. i \int d^{2} x J(x) \mathrm{e}^{\mp i k \cdot x}\right|_{k^{0}=\omega_{k}}
$$

can be deformed into the upper and lower parts of the complex time plane until they hit the singularities $t_{*}$ and $t_{*}^{\prime}$ of the solution, ${ }^{26}$ see figure 14. At large $k$ the integrals receive the dominant contribution near the singularities, and

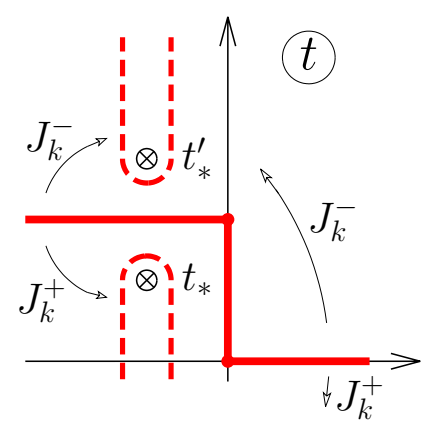

Figure 14. Contours for $J_{k}^{ \pm}$.

$$
J_{k}^{+} \sim J_{0}^{+} \mathrm{e}^{-i \omega_{k} t_{*}}, \quad J_{k}^{-} \sim J_{0}^{-} \mathrm{e}^{i \omega_{k} t_{*}^{\prime}}
$$

where $J_{0}^{ \pm}$are constants.

\footnotetext{
${ }^{26}$ Recall that $J(x)$ is related to $\phi_{0}(x)$.
} 
We finally compute the energy of the initial particles with momentum $k$ in eq. (3.3) using eqs. (B.3), (B.4),

$$
\varepsilon_{k} \equiv \frac{a_{k} b_{k}^{*}}{4 \pi}=\frac{\left|\left(J_{k}^{+}\right)^{*}-J_{k}^{-}\right|^{2}}{16 \pi \sinh ^{2}\left(\omega_{k} T+\theta / 2\right)},
$$

Estimating the largest term in the nominator by eq. (B.6), one obtains the high-frequency asymptotic (4.3) with $T_{*}=T+\min \left(-\operatorname{Im} t_{*}, \operatorname{Im} t_{*}^{\prime}\right)$. Equation (B.7) obeys ${ }^{27}$ the rescaling property (5.2) of section 5 .

Open Access. This article is distributed under the terms of the Creative Commons Attribution License (CC-BY 4.0), which permits any use, distribution and reproduction in any medium, provided the original author(s) and source are credited.

\section{References}

[1] A.A. Belavin, A.M. Polyakov, A.S. Schwartz and Y.S. Tyupkin, Pseudoparticle Solutions of the Yang-Mills Equations, Phys. Lett. B 59 (1975) 85 [inSPIRE].

[2] G. 't Hooft, Computation of the Quantum Effects Due to a Four-Dimensional Pseudoparticle, Phys. Rev. D 14 (1976) 3432 [Erratum ibid. 18 (1978) 2199] [INSPIRE].

[3] V.A. Rubakov and M.E. Shaposhnikov, Electroweak baryon number nonconservation in the early universe and in high-energy collisions, Phys. Usp. 39 (1996) 461 [Usp. Fiz. Nauk 166 (1996) 493] [hep-ph/9603208] [INSPIRE].

[4] I.V. Krive and A.D. Linde, On the Vacuum Stability Problem in Gauge Theories, Nucl. Phys. B 117 (1976) 265 [INSPIRE].

[5] N.V. Krasnikov, Restriction of the Fermion Mass in Gauge Theories of Weak and Electromagnetic Interactions, Yad. Fiz. 28 (1978) 549 [INSPIRE].

[6] G. Degrassi et al., Higgs mass and vacuum stability in the Standard Model at NNLO, JHEP 08 (2012) 098 [arXiv: 1205.6497] [INSPIRE].

[7] D. Buttazzo et al., Investigating the near-criticality of the Higgs boson, JHEP 12 (2013) 089 [arXiv: 1307.3536] [INSPIRE].

[8] F. Bezrukov and M. Shaposhnikov, Why should we care about the top quark Yukawa coupling?, J. Exp. Theor. Phys. 120 (2015) 335 [arXiv:1411.1923] [INSPIRE].

[9] A. Ringwald, High-Energy Breakdown of Perturbation Theory in the Electroweak Instanton Sector, Nucl. Phys. B 330 (1990) 1 [InSPIRE].

[10] O. Espinosa, High-Energy Behavior of Baryon and Lepton Number Violating Scattering Amplitudes and Breakdown of Unitarity in the Standard Model, Nucl. Phys. B 343 (1990) 310 [INSPIRE].

[11] M.P. Mattis, The Riddle of high-energy baryon number violation, Phys. Rept. 214 (1992) 159 [INSPIRE].

[12] I.Y. Kobzarev, L.B. Okun and M.B. Voloshin, Bubbles in Metastable Vacuum, Sov. J. Nucl. Phys. 20 (1975) 644 [Yad. Fiz. 20 (1974) 1229] [INSPIRE].

\footnotetext{
${ }^{27}$ In this case the soft background $\phi_{0}$ and related sources $J_{k}^{ \pm}$are almost independent of $T$.
} 
[13] M. Stone, Semiclassical Methods for Unstable States, Phys. Lett. B 67 (1977) 186 [inSPIRE].

[14] S.R. Coleman, The Fate of the False Vacuum. 1. Semiclassical Theory, Phys. Rev. D 15 (1977) 2929 [Erratum ibid. 16 (1977) 1248] [INSPIRE].

[15] S.R. Coleman, The Uses of Instantons, Subnucl. Ser. 15 (1979) 805 [InSPIRE].

[16] N.S. Manton, Topology in the Weinberg-Salam Theory, Phys. Rev. D 28 (1983) 2019 [INSPIRE].

[17] F.R. Klinkhamer and N.S. Manton, A Saddle Point Solution in the Weinberg-Salam Theory, Phys. Rev. D 30 (1984) 2212 [inSPIRE].

[18] D. Amati, M. Ciafaloni and G. Veneziano, Towards an S-matrix description of gravitational collapse, JHEP 02 (2008) 049 [arXiv:0712.1209] [INSPIRE].

[19] G. Dvali, C. Gomez, R.S. Isermann, D. Lüst and S. Stieberger, Black hole formation and classicalization in ultra-Planckian $2 \rightarrow N$ scattering, Nucl. Phys. B 893 (2015) 187 [arXiv: 1409.7405] [INSPIRE].

[20] V.I. Zakharov, Unitarity constraints on multiparticle weak production, Nucl. Phys. B 353 (1991) 683 [INSPIRE].

[21] V.I. Zakharov, High-energy production of scalar bosons in weak coupling theories, Phys. Rev. Lett. 67 (1991) 3650 [INSPIRE].

[22] V.A. Rubakov, D.T. Son and P.G. Tinyakov, Classical boundary value problem for instanton transitions at high-energies, Phys. Lett. B 287 (1992) 342 [INSPIRE].

[23] M. Maggiore and M.A. Shifman, Nonperturbative processes at high-energies in weakly coupled theories: Multi-instantons set an early limit, Nucl. Phys. B 371 (1992) 177 [InSPIRE].

[24] M.B. Voloshin, Catalyzed decay of false vacuum in four-dimensions, Phys. Rev. D 49 (1994) 2014 [hep-ph/9309237] [INSPIRE].

[25] D.G. Levkov and S.M. Sibiryakov, Induced tunneling in QFT: Soliton creation in collisions of highly energetic particles, Phys. Rev. D 71 (2005) 025001 [hep-th/0410198] [INSPIRE].

[26] D. Levkov and S. Sibiryakov, Real-time instantons and suppression of collision-induced tunneling, JETP Lett. 81 (2005) 53 [Pisma Zh. Eksp. Teor. Fiz. 81 (2005) 60] [hep-th/0412253] [INSPIRE].

[27] G. Veneziano, Bound on reliable one instanton cross-sections, Mod. Phys. Lett. A 7 (1992) 1661 [INSPIRE].

[28] P.G. Tinyakov, Instanton like transitions in high-energy collisions, Int. J. Mod. Phys. A 8 (1993) 1823 [INSPIRE].

[29] V.A. Rubakov, private communication.

[30] S.Y. Khlebnikov, Semiclassical approach to multiparticle production, Phys. Lett. B 282 (1992) 459 [INSPIRE].

[31] D. Diakonov and V. Petrov, Nonperturbative isotropic multiparticle production in Yang-Mills theory, Phys. Rev. D 50 (1994) 266 [hep-ph/9307356] [InSPIRE].

[32] A. Ringwald, An Upper bound on the total cross-section for electroweak baryon number violation, JHEP 10 (2003) 008 [hep-ph/0307034] [INSPIRE].

[33] L.A. Anchordoqui et al., Using cosmic neutrinos to search for non-perturbative physics at the Pierre Auger Observatory, Phys. Rev. D 82 (2010) 043001 [arXiv:1004.3190] [INSPIRE]. 
[34] L.A. Anchordoqui, Ultrahigh Energy Cosmic Rays: Facts, Myths and Legends, doi:10.5170/CERN-2013-003.303 (2011) [arXiv: 1104.0509] [INSPIRE].

[35] MoEDAL collaboration, B. Acharya et al., The Physics Programme Of The MoEDAL Experiment At The LHC, Int. J. Mod. Phys. A 29 (2014) 1430050 [arXiv:1405.7662] [INSPIRE].

[36] F.L. Bezrukov, D. Levkov, C. Rebbi, V.A. Rubakov and P. Tinyakov, Semiclassical study of baryon and lepton number violation in high-energy electroweak collisions, Phys. Rev. D 68 (2003) 036005 [hep-ph/0304180] [INSPIRE].

[37] F.L. Bezrukov, D. Levkov, C. Rebbi, V.A. Rubakov and P. Tinyakov, Suppression of baryon number violation in electroweak collisions: Numerical results, Phys. Lett. B 574 (2003) 75 [hep-ph/0305300] [INSPIRE].

[38] S. Dutta, D.A. Steer and T. Vachaspati, Creating Kinks from Particles, Phys. Rev. Lett. 101 (2008) 121601 [arXiv:0803.0670] [INSPIRE].

[39] T. Romanczukiewicz and Y. Shnir, Oscillon resonances and creation of kinks in particle collisions, Phys. Rev. Lett. 105 (2010) 081601 [arXiv:1002.4484] [INSPIRE].

[40] H. Lamm and T. Vachaspati, Numerical Exploration of Soliton Creation, Phys. Rev. D 87 (2013) 065018 [arXiv:1301.4980] [INSPIRE].

[41] C. Papageorgakis and A.B. Royston, Revisiting Soliton Contributions to Perturbative Amplitudes, JHEP 09 (2014) 128 [arXiv:1404.0016] [INSPIRE].

[42] D.E. Morrissey, T.M.P. Tait and C.E.M. Wagner, Proton lifetime and baryon number violating signatures at the CERN LHC in gauge extended models, Phys. Rev. D 72 (2005) 095003 [hep-ph/0508123] [INSPIRE].

[43] A. Brandenburg, A. Ringwald and A. Utermann, Instantons in Lepton Pair Production, Nucl. Phys. B 754 (2006) 107 [hep-ph/0605234] [INSPIRE].

[44] G. Dvali, G.F. Giudice, C. Gomez and A. Kehagias, UV-Completion by Classicalization, JHEP 08 (2011) 108 [arXiv: 1010.1415] [INSPIRE].

[45] S.V. Demidov and D.G. Levkov, Soliton-antisoliton pair production in particle collisions, Phys. Rev. Lett. 107 (2011) 071601 [arXiv:1103.0013] [INSPIRE].

[46] V.A. Rubakov, D.T. Son and P.G. Tinyakov, Initial state independence of nonperturbative scattering through thin wall bubbles in $(1+1)$-dimensions, Phys. Lett. B 278 (1992) 279 [INSPIRE].

[47] V.G. Kiselev, The False vacuum decay induced by a two particle collision in two-dimensions, Phys. Rev. D 45 (1992) 2929 [inSPIRE].

[48] M.B. Voloshin, Decay Of False Vacuum In (1+1)-dimensions, Yad. Fiz. 42 (1985) 1017 [Sov. J. Nucl. Phys. 42 (1985) 644] [INSPIRE].

[49] K.B. Selivanov and M.B. Voloshin, Destruction Of False Vacuum By Massive Particles, JETP Lett. 42 (1985) 422 [INSPIRE].

[50] M.B. Voloshin and K.G. Selivanov, On Particle Induced Decay of Metastable Vacuum (in Russian), Yad. Fiz. 44 (1986) 1336 [inSPIRE].

[51] P.B. Arnold and M.P. Mattis, Baryon Violation at the SSC? Recent Claims Reexamined, Phys. Rev. D 42 (1990) 1738 [INSPIRE]. 
[52] S.Y. Khlebnikov, V.A. Rubakov and P.G. Tinyakov, Instanton induced cross-sections below the sphaleron, Nucl. Phys. B 350 (1991) 441 [InSPIRE].

[53] P.G. Tinyakov, Multiparticle instanton induced processes and B violation in high-energy collisions, Phys. Lett. B 284 (1992) 410 [InSPIRE].

[54] A.H. Mueller, Comparing two particle and multiparticle initiated processes in the one instanton sector, Nucl. Phys. B 401 (1993) 93 [INSPIRE].

[55] G.F. Bonini, A.G. Cohen, C. Rebbi and V.A. Rubakov, The Semiclassical description of tunneling in scattering with multiple degrees of freedom, Phys. Rev. D 60 (1999) 076004 [hep-ph/9901226] [INSPIRE].

[56] D.G. Levkov, A.G. Panin and S.M. Sibiryakov, Signatures of unstable semiclassical trajectories in tunneling, J. Phys. A 42 (2009) 205102 [arXiv:0811.3391] [INSPIRE].

[57] W.H. Press, S.A. Teukolski, W.T. Vetterling and B.P. Flannery, Numerical Recipes in C: The Art of Scientific Computing, Cambridge University Press (1988).

[58] S.V. Demidov and D.G. Levkov, to be published.

[59] A.N. Kuznetsov and P.G. Tinyakov, False vacuum decay induced by particle collisions, Phys. Rev. D 56 (1997) 1156 [hep-ph/9703256] [INSPIRE].

[60] W.H. Miller, Semiclassical Treatment of Multiple Turning-Point Problems - Phase Shifts and Eigenvalues, J. Chem. Phys. 48 (1968) 1651.

[61] M.J. Davis and E.J. Heller, Quantum dynamical tunneling in bound states, J. Chem. Phys. 75 (1981) 246.

[62] S.V. Demidov and D.G. Levkov, Soliton pair creation in classical wave scattering, JHEP 06 (2011) 016 [arXiv:1103.2133] [INSPIRE].

[63] S.Y. Khlebnikov, V.A. Rubakov and P.G. Tinyakov, Periodic instantons and scattering amplitudes, Nucl. Phys. B 367 (1991) 334 [INSPIRE].

[64] D.S. Gorbunov et al., Valerii Anatol'evich Rubakov (on his 60th birthday), Usp. Fiz. Nauk 185 (2015) 221 [Phys. Usp. 58 (2015) 202]. 